\title{
NONLINEAR FEEDBACK STABILIZATION OF A TWO-DIMENSIONAL BURGERS EQUATION*
}

\author{
Laetitia Thevenet ${ }^{1}$, Jean-Marie Buchot ${ }^{1}$ And Jean-Pierre Raymond ${ }^{1}$
}

\begin{abstract}
In this paper, we study the stabilization of a two-dimensional Burgers equation around a stationary solution by a nonlinear feedback boundary control. We are interested in Dirichlet and Neumann boundary controls. In the literature, it has already been shown that a linear control law, determined by stabilizing the linearized equation, locally stabilizes the two-dimensional Burgers equation. In this paper, we define a nonlinear control law which also provides a local exponential stabilization of the two-dimensional Burgers equation. We end this paper with a few numerical simulations, comparing the performance of the nonlinear law with the linear one.
\end{abstract}

Mathematics Subject Classification. 93B52, 93C20, 93D15.

Received December 2nd, 2008. Revised May 5, 2009.

Published online July 31, 2009.

\section{INTRODUCTION}

In this paper we are interested in the local feedback stabilization of a scalar Burgers type equation, in a two dimensional domain $\Omega$, of the form

$$
\left\{\begin{array}{l}
\partial_{t} z-\nu \Delta z+z \partial_{1} z+z \partial_{2} z=f \quad \text { in } \Omega \times(0, \infty), \\
\nu \frac{\partial z}{\partial n}=g+m u \quad \text { on } \Gamma \times(0, \infty), \\
z(0)=w+y_{0} \quad \text { in } \Omega .
\end{array}\right.
$$

In this setting the symbols $\partial_{1}$ and $\partial_{2}$ denote the partial derivatives with respect to $x_{1}$ and $x_{2}$ respectively, $\nu>0$ is the viscosity coefficient, $u$ is the control, the function $m$ is introduced to localize the control in a part of the boundary $\Gamma=\partial \Omega$, and $w$ is a given stationary solution to equation

$$
-\nu \Delta w+w \partial_{1} w+w \partial_{2} w=f \quad \text { in } \Omega, \quad \nu \frac{\partial w}{\partial n}=g \quad \text { on } \Gamma .
$$

\footnotetext{
Keywords and phrases. Dirichlet control, Neumann control, feedback control, stabilization, Burgers equation, Algebraic Riccati equation.

* This work has been supported by the ANR-project BLAN08-0115-02 CORMORED.

1 Université de Toulouse, UPS, Institut de Mathématiques, UMR CNRS 5219, 31062 Toulouse Cedex 9, France.

Laetitia.Thevenet@math.univ-toulouse.fr; jean-marie.buchot@math.univ-toulouse.fr; raymond@math.univ-toulouse.fr 
We would like to find $u$ in feedback form so that $z-w$ is exponentially stable with a prescribed decay rate $-\alpha<0$, for initial values $y_{0}$ small enough in a space which is specified later on.

We also consider the same type of equation with a Dirichlet boundary control. In both cases, equations satisfied by $y=z-w$ may be written in the abstract form

$$
y^{\prime}=A y+F(y)+B_{m} u, \quad y(0)=y_{0},
$$

where $A$, with domain $D(A)$, is the infinitesimal generator of an analytic semigroup on a Hilbert space $Y, F(y)$ is the nonlinear term in the equation, $B_{m}$ is the operator from a control space $U$ into $Y$ (in the considered problems, $B_{m}$ is an unbounded operator). We assume that the pair $\left(A, B_{m}\right)$ is stabilizable.

Local stabilizability results may be proved for equation (1.3) (see e.g. [16] where stabilizability results are established for the 2D-Navier-Stokes equations instead of a 2D-Burgers equation).

Therefore, if $y_{0}$ is small enough in an appropriate norm, there exist controls $u$ in $L^{2}(0, \infty ; U)$ for which the solution $y_{u}$ to equation (1.3) obeys

$$
J\left(y_{u}, u\right)<\infty \quad \text { where } \quad J\left(y_{u}, u\right)=\frac{1}{2} \int_{0}^{\infty}\left|y_{u}(t)\right|_{Y}^{2} \mathrm{~d} t+\frac{1}{2} \int_{0}^{\infty}|u(t)|_{U}^{2} \mathrm{~d} t .
$$

Such a control can be obtained by solving the nonlinear closed loop system with a linear feedback law of the form $u=-B_{m}^{*} \Pi y$, where $\Pi$ is the solution to the algebraic Riccati equation of a LQR problem. This is the way followed in [16]. In that case, we do not take into account the nonlinearity of the model in the feedback law. One way to obtain another control, possibly more efficient or more robust, able to stabilize equation (1.3), could be to look for a solution to the control problem

$$
\inf \{J(y, u) \mid(y, u) \text { is solution of }(1.3)\} .
$$

When $B_{m}$ is a bounded operator, it can be shown that this problem admits a solution (provided that $y_{0}$ is small enough). If the Hamilton-Jacobi-Bellman equation

$$
\langle A y+F(y), \mathcal{G}(y)\rangle-\frac{1}{2}\left|B_{m}^{*} \mathcal{G}(y)\right|_{U}^{2}+\frac{1}{2}|y|_{Y}^{2}=0, \quad \text { for all } y \in D(A),
$$

admits a solution $\mathcal{G}$, then it can be used to determine a solution to $(\mathcal{P})$ and the corresponding control in feedback form (the gradient of the value function of $(\mathcal{P})$ may provide a solution to equation (1.4)). But in the case when $B_{m}$ is an unbounded operator, equation (1.4) is not well posed since the nonlinear term $B_{m}^{*} \mathcal{G}(y)$ is not well defined.

The main objective of this paper is to investigate an intermediate way (intermediate between the linear feedback law and the nonlinear law determined by solving equation (1.4)). We are going to see that even if equation (1.4) is not necessarily well posed, it is possible to find a nonlinear feedback law, obtained by using a power series expansion method, which is a formal approximate solution to the Hamilton-Jacobi-Bellman equation (1.4). This kind of method is well known in the case of systems governed by ordinary differential equations (see [3]). Let us explain how we can obtain such a nonlinear feedback law in the case of equation (1.1). The algebraic Riccati equation associated with $J$ and with the linearized equation corresponding to (1.3) is

$$
\Pi=\Pi^{*} \geq 0, \quad \Pi A+A^{*} \Pi-\Pi B_{m} B_{m}^{*} \Pi+I=0
$$

(see (2.5) for a more precise statement). This equation is equivalent to

$$
\Pi=\Pi^{*} \geq 0, \quad\langle A y, \Pi y\rangle-\frac{1}{2}\left|B_{m}^{*} \Pi y\right|_{U}^{2}+\frac{1}{2}|y|_{Y}^{2}=0, \quad \text { for all } y \in D(A),
$$


which corresponds to $(1.4)$ when $F(y)=0$ and $\mathcal{G}(y)=\Pi y$. Now, let us write a formal Taylor expansion of $\mathcal{G}$ about 0

$$
\mathcal{G}(y)=\mathcal{G}(0)+\mathcal{G}^{\prime}(0) y+\frac{1}{2} \mathcal{G}^{\prime \prime}(0)(y, y)+\ldots
$$

By substituting (1.5) into (1.4) and by identifying the terms having the same order with respect to $y$ we obtain

(a) $\left|B_{m}^{*} \mathcal{G}(0)\right|_{U}^{2}=0$ for the order 0

(b) $\langle A y, \mathcal{G}(0)\rangle=0$ for the order 1 ;

(c) $\left\langle A y, \mathcal{G}^{\prime}(0) y\right\rangle+\langle F(y), \mathcal{G}(0)\rangle-\frac{1}{2}\left|B_{m}^{*} \mathcal{G}^{\prime}(0) y\right|_{U}^{2}+\frac{1}{2}|y|_{Y}^{2}=0$ for the order 2

(d) $\frac{1}{2}\left\langle A y, \mathcal{G}^{\prime \prime}(0)(y, y)\right\rangle-\frac{1}{2}\left\langle B_{m} B_{m}^{*} \mathcal{G}^{\prime}(0) y, \mathcal{G}^{\prime \prime}(0)(y, y)\right\rangle+\left\langle F(y), \mathcal{G}^{\prime}(0) y\right\rangle=0$ for the order 3 .

We notice that

$$
\mathcal{G}(0)=0, \quad \mathcal{G}^{\prime}(0)=\Pi \quad \text { and } \quad \mathcal{G}^{\prime \prime}(0)(y, y)=-2 A_{\Pi}^{-*} \Pi F(y)
$$

where $A_{\Pi}^{-*}$ is the inverse of $A_{\Pi}^{*}$ (the adjoint of $A_{\Pi}=A-B_{m} B_{m}^{*} \Pi$ ), is a triplet satisfying $(a),(b),(c)$ and $(d)$. This is why we choose

$$
u=-B_{m}^{*} \Pi y+B_{m}^{*} A_{\Pi}^{-*} \Pi F(y)
$$

as nonlinear feedback control law to stabilize equation (1.3). We prove that this feedback guarantees a local stabilizability of the closed loop system. Though our approach is quite general and may be applied to various systems, we only study it in the case of a two dimensional Burgers type equation with a control applied either in a Dirichlet or a Neumann boundary condition. The case of the Navier-Stokes equations will be investigated in a forthcoming paper.

The plan of this paper is as follows. We first study the case of a Neumann boundary control in Sections 2-6. The adaptation to the case of a Dirichlet boundary control is performed in Section 7 . We describe the precise setting of our problem in Section 2. The properties of operators $A, A^{*}, B_{m}, B_{m}^{*}$, $\Pi$ and $A_{\Pi}$ are briefly recalled in Section 3. In order to study the nonlinear closed loop system, we first establish a regularity result for a nonhomogeneous closed loop linear equation in Section 4. The main result of the paper is stated in Theorem 5.1 and its proof is given in Section 5. We explain how to adapt the results of Section 6 to obtain a prescribed decay rate. In Section 8, we present some numerical tests where we compare the linear and the nonlinear feedback laws applied to the nonlinear system. Though there is no conclusion valid for all numerical tests, we present examples for which the nonlinear feedback law is able to stabilize the nonlinear system while the linear feedback law, with the same initial condition, is not able. We can observe that the behaviors of the solution of the closed loop system with the linear and the nonlinear feedback laws are quite different.

Let us finally mention that many papers deal with the stabilization of the one dimensional viscous Burgers equation. We refer to [11] for recent results in that direction (see also the references therein).

\section{Setting of the Problem}

Throughout the following, $\Omega$ is either a rectangle in $\mathbb{R}^{2}$ or a bounded domain in $\mathbb{R}^{2}$ with a boundary $\Gamma$ of class $C^{3}$. If one of these geometrical assumptions is satisfied, it can be shown that the solution to the second order elliptic equations we consider, with homogeneous Neumann or Dirichlet boundary conditions, belongs to $H^{s+2}(\Omega)$ if the right hand side belongs to $H^{s}(\Omega)$ with $0 \leq s<1 / 2$. We set $\Sigma_{\infty}=\Gamma \times(0, \infty)$, and $Q_{\infty}=\Omega \times(0, \infty)$.

We make the following assumptions on the function $m$. When $\Omega$ is a domain with a boundary $\Gamma$ of class $C^{3}$, we assume that $m \in C^{2}(\Gamma), m \geq 0$, and $m(x)=1$ for all $x \in \Gamma_{c}$, where $\Gamma_{c}$ is a nonempty open subset in $\Gamma$.

When $\Omega=] 0, a[\times] 0, b\left[\right.$ is a rectangle, we assume that $m \in C^{2}\left(\Gamma_{a}\right), \Gamma_{a}=\{a\} \times[0, b], m \geq 0$, and $m(x)=1$ for all $x \in \Gamma_{c}, \Gamma_{c}$ is a nonempty segment in $\Gamma_{a}$. In the case of a Neumann boundary control, $\Gamma_{c}$ can be either 
a segment in $\Gamma_{a}$ satisfying $\overline{\Gamma_{c}} \subset \Gamma_{a}$ or $\overline{\Gamma_{c}}=\Gamma_{a}$, and in that case $m(x)=1$ for all $x \in \Gamma_{a}$. In the case of a Dirichlet boundary control, we assume that $m$ belongs to $C^{2}\left(\Gamma_{a}\right) \cap H_{0}^{2}\left(\Gamma_{a}\right)$.

As indicated in the introduction, we first consider the case of a Neumann boundary control. We assume that the solution $w$ to equation (1.2) belongs to $H^{3}(\Omega)$. If $z$ is the solution to equation (1.1), then $y=z-w$ obeys

$$
\left\{\begin{array}{l}
\partial_{t} y-\nu \Delta y+y\left(\partial_{1} w+\partial_{2} w\right)+w\left(\partial_{1} y+\partial_{2} y\right)+y\left(\partial_{1} y+\partial_{2} y\right)=0 \text { in } Q_{\infty}, \\
\nu \frac{\partial y}{\partial n}=m u \quad \text { on } \Sigma_{\infty} \\
y(0)=y_{0} \quad \text { in } \Omega .
\end{array}\right.
$$

We denote by $(A, D(A))$ and $\left(A^{*}, D\left(A^{*}\right)\right)$ the unbounded operators in $L^{2}(\Omega)$ defined by

$$
\begin{array}{ll}
D(A)=\left\{y \in H^{2}(\Omega) \mid \nu \frac{\partial y}{\partial n}=0 \text { on } \Gamma\right\}, & A y=\nu \Delta y-y\left(\partial_{1} w+\partial_{2} w\right)-w\left(\partial_{1} y+\partial_{2} y\right), \\
D\left(A^{*}\right)=\left\{y \in H^{2}(\Omega) \mid \nu \frac{\partial y}{\partial n}+y w=0 \text { on } \Gamma\right\}, & A^{*} y=\nu \Delta y+w\left(\partial_{1} y+\partial_{2} y\right) .
\end{array}
$$

Since $w \in H^{3}(\Omega)$, we can easily verify that there exists $\lambda_{0}>0$ in the resolvent set of $A$ satisfying

and

$$
\left(\left(\lambda_{0} I-A\right) y, y\right)_{L^{2}(\Omega)} \geq \frac{\nu}{2}|y|_{H^{1}(\Omega)}^{2} \quad \text { for all } y \in D(A)
$$

$$
\left(\left(\lambda_{0} I-A^{*}\right) y, y\right)_{L^{2}(\Omega)} \geq \frac{\nu}{2}|y|_{H^{1}(\Omega)}^{2} \quad \text { for all } y \in D\left(A^{*}\right) .
$$

Here $|\cdot|_{H^{1}(\Omega)}$ denotes the usual norm in $H^{1}(\Omega)$. We shall use the same type of notation for other spaces. Following [6], Chapter 2, Part II, we are going to rewrite equation (2.1) as an evolution equation. To this aim, we introduce $N \in \mathcal{L}\left(L^{2}(\Gamma), H^{3 / 2}(\Omega)\right)$, the Neumann operator associated with $\lambda_{0} I-A$, defined by $N u=y$, where $y$ is the unique solution in $H^{3 / 2}(\Omega)$ to equation

$$
\lambda_{0} y-\nu \Delta y+w\left(\partial_{1} y+\partial_{2} y\right)+y\left(\partial_{1} w+\partial_{2} w\right)=0 \quad \text { in } \Omega, \quad \nu \frac{\partial y}{\partial n}=u \quad \text { on } \Gamma .
$$

The nonlinear term $-y\left(\partial_{1} y+\partial_{2} y\right)$, which is equal to $-\partial_{1}\left(y^{2} / 2\right)-\partial_{2}\left(y^{2} / 2\right)$, is rewritten as an element $F(y)$ in $\left(D\left(A^{*}\right)\right)^{\prime}$ as follows

$$
\langle F(y), \Phi\rangle_{\left(D\left(A^{*}\right)\right)^{\prime}, D\left(A^{*}\right)}=\frac{1}{2} \int_{\Omega} y^{2}\left(\partial_{1} \Phi+\partial_{2} \Phi\right)-\frac{1}{2} \int_{\Gamma} y^{2}\left(n_{1}+n_{2}\right) \Phi \text { for all } \Phi \in D\left(A^{*}\right),
$$

where $n=\left(n_{1}, n_{2}\right)^{T}$ denotes the unit normal to $\Gamma$ outward $\Omega$. Let us observe that $F(y)$ is well defined in $\left(D\left(A^{*}\right)\right)^{\prime}$ for all $y \in H^{1}(\Omega)$. Setting $B=\left(\lambda_{0} I-A\right) N$, equation (2.1) may be rewritten in the form

$$
y^{\prime}=A y+B M u+F(y) \quad \text { in }(0, \infty), \quad y(0)=y_{0},
$$

where $M$ is the multiplication operator defined by $M u=m u$. The operator $B_{m}$ in the introduction is nothing else than $B_{m}=B M$. As mentioned in the introduction, a way to look for a feedback control able to locally stabilize the nonlinear equation (2.3) is to look for a feedback control stabilizing the linearized equation (see [16])

$$
y^{\prime}=A y+B M u \quad \text { in }(0, \infty), \quad y(0)=y_{0} .
$$

The stabilizability of the pair $(A, B M)$ may be proved thanks to the null controllability result proved in [8] for a similar equation with a distributed control. A feedback control stabilizing equation (2.4) can be determined 
by solving a Linear-Quadratic-Regulation problem with the identity as observation operator. In that case the feedback is obtained by solving the algebraic Riccati equation

$$
\begin{aligned}
& \Pi=\Pi^{*} \in \mathcal{L}\left(L^{2}(\Omega)\right), \quad \Pi \geq 0, \\
& |\Pi y|_{D\left(A^{*}\right)} \leq|y|_{L^{2}(\Omega)} \quad \text { for all } y \in L^{2}(\Omega), \\
& \Pi A+A^{*} \Pi-\Pi B M^{2} B^{*} \Pi+I=0 .
\end{aligned}
$$

The existence of a unique weak solution to the above algebraic Riccati equation may be deduced from [12], while the estimate in the second line of (2.5) can be proved as in [16], Theorem 4.5. In Lemma 3.3, we show that $\Pi$ may be extended as an operator belonging to $\mathcal{L}\left(\left(D\left(A^{*}\right)\right)^{\prime}, L^{2}(\Omega)\right)$.

We would like to study the following nonlinear feedback law

$$
u=-M B^{*} \Pi y+M B^{*} A_{\Pi}^{-*} \Pi F(y),
$$

where $\Pi$ is the solution to equation (2.5) and $A_{\Pi}=A-B M^{2} B^{*} \Pi$. That means that we have to study the following equation

$$
y^{\prime}=A_{\Pi} y+B M^{2} B^{*} A_{\Pi}^{-*} \Pi F(y)+F(y) \quad \text { in }(0, \infty), \quad y(0)=y_{0} .
$$

To study such an equation, we shall consider the nonhomogeneous equation

$$
y^{\prime}=A_{\Pi} y+f+B M g \quad \text { in }(0, \infty), \quad y(0)=y_{0},
$$

where $f$ and $g$ will play the role of $F(y)$ and of $M B^{*} A_{\Pi}^{-*} \Pi F(y)$. Next, we shall use a fixed point method to prove the existence of a solution to equation (2.6). Throughout the following, we use $C, C_{1}, \ldots, C_{4}$ to denote various constants depending on $\Omega$ and $w$. They also may depend on some small parameter $\varepsilon$.

\section{Properties of operators $N, B, \Pi, A_{\Pi}$ And their adjoints}

Let us first recall well known results which will be useful in what follows.

Theorem 3.1. The unbounded operator $\left(A-\lambda_{0} I\right)$ (respectively $\left(A^{*}-\lambda_{0} I\right)$ ) with domain $D\left(A-\lambda_{0} I\right)=D(A)$ (respectively $\left.D\left(A^{*}-\lambda_{0} I\right)=D\left(A^{*}\right)\right)$ is the infinitesimal generator of a bounded analytic semigroup on $L^{2}(\Omega)$. Moreover, for all $0 \leq \alpha \leq 1$, we have

$$
\begin{gathered}
D\left(\left(\lambda_{0} I-A\right)^{\alpha}\right)=D\left(\left(\lambda_{0} I-A^{*}\right)^{\alpha}\right)=H^{2 \alpha}(\Omega) \quad \text { if } \alpha \in[0,3 / 4[ \\
\left.\left.D\left(\left(\lambda_{0} I-A\right)^{\alpha}\right)=\left\{z \in H^{2 \alpha}(\Omega) \mid \nu \frac{\partial z}{\partial n}=0 \text { on } \Gamma\right\} \quad \text { if } \alpha \in\right] 3 / 4,1\right],
\end{gathered}
$$

and

$$
\left.\left.D\left(\left(\lambda_{0} I-A^{*}\right)^{\alpha}\right)=\left\{z \in H^{2 \alpha}(\Omega) \mid \nu \frac{\partial z}{\partial n}+z w=0 \text { on } \Gamma\right\} \quad \text { if } \alpha \in\right] 3 / 4,1\right] .
$$

Proof. Under condition (2.2) the analyticity of the semigroup generated by $\left(A-\lambda_{0} I\right)$ is well known ([5], Chap. 1 , Thm. 2.12). The characterization of the domains of fractional powers of $\left(\lambda_{0} I-A\right)$ and $\left(\lambda_{0} I-A^{*}\right)$ may be deduced from [14].

Observe that the semigroups $\left(\mathrm{e}^{t\left(A-\lambda_{0} I\right)}\right)_{t \geq 0}$ and $\left(\mathrm{e}^{t\left(A^{*}-\lambda_{0} I\right)}\right)_{t \geq 0}$ are exponentially stable on $L^{2}(\Omega)$ and that

$$
\left\|\mathrm{e}^{t\left(A-\lambda_{0} I\right)}\right\|_{\mathcal{L}\left(L^{2}(\Omega)\right)} \leq \mathrm{e}^{-\beta t} \text { and }\left\|\mathrm{e}^{t\left(A^{*}-\lambda_{0} I\right)}\right\|_{\mathcal{L}\left(L^{2}(\Omega)\right)} \leq \mathrm{e}^{-\beta t},
$$

for all $\beta<\nu / 2$ (see [5], Chap. 1, Thm. 2.12). 
In the following, it is useful to introduce the notation

$$
\mathcal{H}^{\theta}(\Omega)=D\left(\left(\lambda_{0} I-A^{*}\right)^{\theta / 2}\right) \quad \text { and } \quad \mathcal{H}^{-\theta}(\Omega)=\left(\mathcal{H}^{\theta}(\Omega)\right)^{\prime} \quad \text { for } \quad 0 \leq \theta \leq 2 .
$$

Lemma 3.1. (i) The operator $N \in \mathcal{L}\left(L^{2}(\Gamma), L^{2}(\Omega)\right)$ satisfies

$$
|N u|_{H^{3 / 2}(\Omega)} \leq C|u|_{L^{2}(\Gamma)} .
$$

(ii) The operator $N^{*} \in \mathcal{L}\left(L^{2}(\Omega), L^{2}(\Gamma)\right)$, the adjoint operator of $N \in \mathcal{L}\left(L^{2}(\Gamma), L^{2}(\Omega)\right)$, is defined by

$$
N^{*} g=\left.z\right|_{\Gamma}
$$

where $z$ is the solution of

$$
\lambda_{0} z-\nu \Delta z-w\left(\partial_{1} z+\partial_{2} z\right)=g \quad \text { in } \Omega, \quad \nu \frac{\partial z}{\partial n}+w z=0 \quad \text { on } \Gamma .
$$

Proof. The proof of $(i)$ is classical, it relies on regularity results for elliptic equations. The proof of $(i i)$ relies on Green formula.

The operator $B=\left(\lambda_{0} I-A\right) N$ can be considered either as an unbounded operator from $L^{2}(\Gamma)$ into $L^{2}(\Omega)$ or as a bounded operator from $L^{2}(\Gamma)$ into $\left(D\left(A^{*}\right)\right)^{\prime}$. Thus $B^{*}$ can be considered as a bounded operator from $D\left(A^{*}\right)$ into $L^{2}(\Gamma)$.

Proposition 3.1. For all $\Phi \in D\left(A^{*}\right), B^{*} \Phi$ is defined by

$$
B^{*} \Phi=N^{*}\left(\lambda_{0} I-A^{*}\right) \Phi=\left.\Phi\right|_{\Gamma}
$$

and we have

$$
\left|M B^{*} \Phi\right|_{H^{s-1 / 2}(\Gamma)} \leq C|\Phi|_{H^{s}(\Omega)} \quad \text { if } 1 / 2<s \leq 2 .
$$

Proof. The proof directly follows from the expression obtained for $N^{*}$.

Lemma 3.2. The solution $\Pi \in \mathcal{L}\left(L^{2}(\Omega)\right)$ to the algebraic Riccati equation (2.5) may be extended as a linear and continuous operator from $\left(D\left(A^{*}\right)\right)^{\prime}$ into $L^{2}(\Omega)$.

Proof. Let $z$ belong to $\left(D\left(A^{*}\right)\right)^{\prime}$ and $\left(z_{n}\right)_{n} \subset L^{2}(\Omega)$ be such that $z_{n} \rightarrow z$ in $\left(D\left(A^{*}\right)\right)^{\prime}$. For all $y$ in $L^{2}(\Omega)$, since $\Pi$ belongs to $\mathcal{L}\left(L^{2}(\Omega), D\left(A^{*}\right)\right)$, we have

$$
\left|\left(\Pi y, z_{n}\right)_{L^{2}(\Omega)}\right|=\left|\left\langle\Pi y, z_{n}\right\rangle_{D\left(A^{*}\right),\left(D\left(A^{*}\right)\right)^{\prime}}\right| \leq C\left|z_{n}\right|_{\left(D\left(A^{*}\right)\right)^{\prime}}|y|_{L^{2}(\Omega)} .
$$

Using $\Pi=\Pi^{*}$, we obtain

Thus

$$
\left|\left(y, \Pi z_{n}\right)_{L^{2}(\Omega)}\right| \leq C\left|z_{n}\right|_{\left(D\left(A^{*}\right)\right)^{\prime}}|y|_{L^{2}(\Omega)} .
$$

$$
\left|\Pi z_{n}\right|_{L^{2}(\Omega)} \leq C\left|z_{n}\right|_{\left(D\left(A^{*}\right)\right)^{\prime}} .
$$

Passing to the limit in the above estimate, we obtain that $\Pi \in \mathcal{L}\left(\left(D\left(A^{*}\right)\right)^{\prime}, L^{2}(\Omega)\right)$.

Following $[1,16]$, we can define $\left(A_{\Pi}, D\left(A_{\Pi}\right)\right)$ by

$$
D\left(A_{\Pi}\right)=\left\{y \in L^{2}(\Omega) \mid A y-B M^{2} B^{*} \Pi y \in L^{2}(\Omega)\right\}=\left\{y \in H^{2}(\Omega) \mid \nu \frac{\partial y}{\partial n}+M^{2} B^{*} \Pi y=0 \text { on } \Gamma\right\},
$$

and

$$
A_{\Pi} y=A y-B M^{2} B^{*} \Pi y \quad \text { for all } y \in D\left(A_{\Pi}\right) .
$$


Lemma 3.3. $(i)$ The operator $\left(A_{\Pi}, D\left(A_{\Pi}\right)\right)$ is the infinitesimal generator of an analytic semigroup exponentially stable on $L^{2}(\Omega)$. The adjoint of the unbounded operator $\left(A_{\Pi}, D\left(A_{\Pi}\right)\right)$ in $L^{2}(\Omega)$ is defined by

$$
D\left(A_{\Pi}^{*}\right)=D\left(A^{*}\right), \quad A_{\Pi}^{*} \Phi=A^{*} \Phi-\left(B^{*} \Pi\right)^{*} M^{2} B^{*} \Phi \quad \text { for all } \Phi \in D\left(A_{\Pi}^{*}\right) .
$$

(ii) $A_{\Pi}^{*}$ is bijective from $D\left(A^{*}\right)$ into $L^{2}(\Omega)$.

Proof. For $(i)$, see e.g. [16]. To prove (ii) it is enough to observe that $A_{\Pi}^{*} y=f \in L^{2}(\Omega)$ if and only if

$$
y=-\int_{0}^{\infty} \mathrm{e}^{\tau A_{\Pi}^{*}} f \mathrm{~d} \tau \in D\left(A_{\Pi}^{*}\right) .
$$

\section{Nonhomogeneous EQUations}

In the following, we shall use the notations

$$
H^{s, r}\left(Q_{\infty}\right)=L^{2}\left(0, \infty ; H^{s}(\Omega)\right) \cap H^{r}\left(0, \infty ; L^{2}(\Omega)\right) \text { and } H^{s, r}\left(\Sigma_{\infty}\right)=L^{2}\left(0, \infty ; H^{s}(\Gamma)\right) \cap H^{r}\left(0, \infty ; L^{2}(\Gamma)\right)
$$

Let us first recall a regularity result for the equation

$$
y^{\prime}=\left(A-\lambda_{0} I\right) y+B M h, \quad y(0)=0,
$$

where $\lambda_{0}$ is chosen so that (2.2) is satisfied.

Lemma 4.1. If $h \in H^{s, s / 2}\left(\Sigma_{\infty}\right)$ with $0 \leq s<3 / 2, s \neq 1$, and $h(\cdot, 0)=0$ on $\Gamma$ when $s>1$, then the solution of equation (4.1) obeys

$$
\|y\|_{H^{s+3 / 2, s / 2+3 / 4}\left(Q_{\infty}\right)} \leq C\|h\|_{H^{s, s / 2}\left(\Sigma_{\infty}\right)} .
$$

Proof. See [15], Chapter 4, Part 13.

We now study the equation

$$
y^{\prime}=A_{\Pi} y+f+B M g, \quad y(0)=y_{0} .
$$

We recall the following isomorphism lemma that we will use later on.

Lemma 4.2. Let $Y$ be a Hilbert space and suppose that $A$ is the infinitesimal generator of an analytic semigroup of negative type on $Y$. Then, the mapping

$$
\begin{array}{ccc}
L^{2}(0, \infty ; Y) \cap H^{1}\left(0, \infty ;\left(D\left(A^{*}\right)\right)^{\prime}\right) & \mapsto & L^{2}\left(0, \infty ; D\left(A^{*}\right)^{\prime}\right) \times\left[D\left(A^{*}\right), Y\right]_{1 / 2}^{\prime} \\
y & \mapsto & \left(y^{\prime}-A y, y(0)\right)
\end{array}
$$

is an isomorphism.

Proof. The proof is a direct consequence of [5], Chapters 1-3.

Theorem 4.1. If $f \in L^{2}\left(0, \infty ; \mathcal{H}^{-1+\varepsilon}(\Omega)\right), g \in L^{2}\left(\Sigma_{\infty}\right), y_{0} \in H^{\varepsilon}(\Omega)$ with $0 \leq \varepsilon<1 / 2$, then equation (4.2) admits a unique solution in $L^{2}\left(0, \infty ; L^{2}(\Omega)\right) \cap H^{1}\left(0, \infty ;\left(D\left(A^{*}\right)\right)^{\prime}\right)$ which obeys

$$
\|y\|_{H^{1+\varepsilon, 1 / 2+\varepsilon / 2}\left(Q_{\infty}\right)} \leq C_{1}\left(\left|y_{0}\right|_{H^{\varepsilon}(\Omega)}+\|f\|_{L^{2}\left(0, \infty ; \mathcal{H}^{-1+\varepsilon}(\Omega)\right)}+\|g\|_{L^{2}\left(\Sigma_{\infty}\right)}\right) .
$$

Proof. We clearly have $f+B M g \in L^{2}\left(0, \infty ;\left(D\left(A_{\Pi}^{*}\right)\right)^{\prime}\right)=L^{2}\left(0, \infty ;\left(D\left(A^{*}\right)\right)^{\prime}\right)$ since $D\left(A_{\Pi}^{*}\right)=D\left(A^{*}\right)$. Moreover, $y_{0} \in H^{\varepsilon}(\Omega) \subset\left[D\left(A^{*}\right), L^{2}(\Omega)\right]_{1 / 2}^{\prime}$. We can apply Lemma 4.2. Thus the solution to equation (4.2) obeys

$$
\|y\|_{L^{2}\left(0, \infty ; L^{2}(\Omega)\right)} \leq C\left(\left|y_{0}\right|_{H^{\varepsilon}(\Omega)}+\|f\|_{L^{2}\left(0, \infty ; \mathcal{H}^{-1+\varepsilon}(\Omega)\right)}+\|g\|_{L^{2}\left(\Sigma_{\infty}\right)}\right)
$$


Let us show that this solution belongs to $H^{1+\varepsilon, 1 / 2+\varepsilon / 2}\left(Q_{\infty}\right)$ and obeys the estimate (4.3). Due to the definition of $A_{\Pi}, y$ is solution of

$$
y^{\prime}=A y+f+B M\left(g-M B^{*} \Pi y\right), \quad y(0)=y_{0} .
$$

We set $y=y_{1}+y_{2}$ where $y_{1}$ is solution to

$$
y_{1}^{\prime}=\left(A-\lambda_{0} I\right) y_{1}+\tilde{f}, \quad y_{1}(0)=y_{0},
$$

with $\tilde{f}=f+\lambda_{0} y \in L^{2}\left(0, \infty ; \mathcal{H}^{-1+\varepsilon}(\Omega)\right)$, and $y_{2}$ is solution to

$$
y_{2}^{\prime}=\left(A-\lambda_{0} I\right) y_{2}+B M h, \quad y_{2}(0)=0,
$$

with $h=g-M B^{*} \Pi y$. Let us first estimate $y_{1}$. We can check that

$$
H^{\varepsilon}(\Omega)=\left[\left[D\left(A^{*}\right), L^{2}(\Omega)\right]_{1 / 2}^{\prime},\left[L^{2}(\Omega), D(A)\right]_{1 / 2}\right]_{(1+\varepsilon) / 2} .
$$

Moreover,

$$
\tilde{f} \in L^{2}\left(0, \infty ; \mathcal{H}^{-1+\varepsilon}(\Omega)\right)=L^{2}\left(0, \infty ;\left[D\left(A^{*}\right), L^{2}(\Omega)\right]_{(1+\varepsilon) / 2}^{\prime}\right) .
$$

By interpolation, with Lemma 4.2 and [5], Chapter 3, it follows that

$$
y_{1} \in L^{2}\left(0, \infty ;\left[L^{2}(\Omega), D(A)\right]_{(1+\varepsilon) / 2}\right) \cap H^{1}\left(0, \infty ;\left[D\left(A^{*}\right), L^{2}(\Omega)\right]_{(1+\varepsilon) / 2}^{\prime}\right) .
$$

As $\left[L^{2}(\Omega), D(A)\right]_{(1+\varepsilon) / 2} \subset H^{1+\varepsilon}(\Omega)$, we clearly obtain $y_{1} \in L^{2}\left(0, \infty ; H^{1+\varepsilon}(\Omega)\right)$. Finally, by interpolation $y_{1} \in$ $H^{1 / 2+\varepsilon / 2}\left(0, \infty ; L^{2}(\Omega)\right)$ and

$$
\begin{aligned}
\left\|y_{1}\right\|_{H^{1+\varepsilon, 1 / 2+\varepsilon / 2}\left(Q_{\infty}\right)} & \leq C\left(\left|y_{0}\right|_{H^{\varepsilon}(\Omega)}+\|f\|_{L^{2}\left(0, \infty ; \mathcal{H}^{-1+\varepsilon}(\Omega)\right)}+\|y\|_{L^{2}\left(0, \infty ; L^{2}(\Omega)\right)}\right) \\
& \leq C\left(\left|y_{0}\right|_{H^{\varepsilon}(\Omega)}+\|f\|_{L^{2}\left(0, \infty ; \mathcal{H}^{-1+\varepsilon}(\Omega)\right)}+\|g\|_{L^{2}\left(\Sigma_{\infty}\right)}\right) .
\end{aligned}
$$

Since $h$ belongs to $L^{2}\left(\Sigma_{\infty}\right)$, due to Lemma $4.1, y_{2}$ belongs to $H^{1+\varepsilon, 1 / 2+\varepsilon / 2}\left(Q_{\infty}\right)$ and we have

$$
\left\|y_{2}\right\|_{H^{1+\varepsilon, 1 / 2+\varepsilon / 2}\left(Q_{\infty}\right)} \leq C\left(\|g\|_{L^{2}\left(\Sigma_{\infty}\right)}+\|y\|_{L^{2}\left(Q_{\infty}\right)}\right) .
$$

The proof is complete.

\section{Stabilization of the two Dimensional Burgers equation}

In this section, we prove a stabilization result for which the exponential decay is not prescribed. We shall explain in Section 6 how to adapt this result to obtain the exponential decay rate.

Let us consider the Burgers equation with the nonlinear feedback law

$$
y^{\prime}=A_{\Pi} y+B M^{2} B^{*} A_{\Pi}^{-*} \Pi F(y)+F(y), \quad y(0)=y_{0} .
$$

Theorem 5.1. Let $\varepsilon$ belong to the interval $] 1 / 4,1 / 2\left[\right.$. There exist $\mu_{0}>0$ and a nondecreasing function $\eta$ from $\mathbb{R}^{+}$into itself, such that if $\mu \in\left(0, \mu_{0}\right)$ and $\left|y_{0}\right|_{H^{\varepsilon}(\Omega)} \leq \eta(\mu)$, then equation (5.1) admits a unique solution in the set

$$
D_{\mu}=\left\{y \in H^{1+\varepsilon, 1 / 2+\varepsilon / 2}\left(Q_{\infty}\right) \mid\|y\|_{H^{1+\varepsilon, 1 / 2+\varepsilon / 2}\left(Q_{\infty}\right)} \leq \mu\right\} .
$$

To prove this result, we use a fixed point theorem and we consider the equation

$$
y^{\prime}=A_{\Pi} y+F(z)+B M^{2} B^{*} A_{\Pi}^{-*} \Pi F(z), \quad y(0)=y_{0},
$$


for all $z$ in $D_{\mu}$. Equation (5.2) is nothing else than equation (4.2) with

$$
f=F(z), \quad g=M B^{*} A_{\Pi}^{-*} \Pi F(z) .
$$

In the first subsection, we estimate $F(z)$ and $G(z)=M B^{*} A_{\Pi}^{-*} \Pi F(z)$ when $z$ belongs to $D_{\mu}$.

\subsection{Analysis of $F(z)$ and $G(z)$}

Lemma 5.1. Let $\varepsilon$ belong to the interval $] 1 / 4,1 / 2\left[\right.$. If $z$ belongs to $H^{1+\varepsilon, 1 / 2+\varepsilon / 2}\left(Q_{\infty}\right)$ then $F(z) \in L^{2}(0, \infty$; $\left.\mathcal{H}^{-1+\varepsilon}(\Omega)\right)$ and we have

$$
\|F(z)\|_{L^{2}\left(0, \infty ; \mathcal{H}^{-1+\varepsilon}(\Omega)\right)} \leq C_{2}\|z\|_{H^{1+\varepsilon, 1 / 2+\varepsilon / 2}\left(Q_{\infty}\right)}^{2} .
$$

(The constant $C_{2}$ depends on $\varepsilon$.)

Proof. Step 1. Let us first show that

$$
\left|\partial_{i} \Phi\right|_{\mathcal{H}^{-\varepsilon}(\Omega)} \leq C|\Phi|_{\mathcal{H}^{1-\varepsilon}(\Omega)}, \quad i=1,2 \quad \text { for all } \Phi \in H^{1}(\Omega)
$$

We know that $\partial_{i}$ is linear continuous from $H^{1}(\Omega)$ to $L^{2}(\Omega)$. The operator $\partial_{i}$ may be extended as a bounded linear operator from $L^{2}(\Omega)$ to $H^{-1}(\Omega)$ by the formula

$$
\left\langle\partial_{i} \Phi, y\right\rangle_{H^{-1}(\Omega), H_{0}^{1}(\Omega)}=-\int_{\Omega} \Phi \partial_{i} y \quad \text { for all } y \in H_{0}^{1}(\Omega) .
$$

By interpolation $\partial_{i}$ is linear and continuous from $\mathcal{H}^{1-\varepsilon}(\Omega)$ to $\mathcal{H}^{-\varepsilon}(\Omega)$ if $\varepsilon \in[0,1 / 2[$.

Step 2. By definition, for all $\Phi \in L^{2}\left(0, \infty ; H^{1}(\Omega)\right)$, we have

$$
\int_{0}^{\infty}\langle F(z), \Phi\rangle_{\mathcal{H}^{-1+\varepsilon}(\Omega), \mathcal{H}^{1-\varepsilon}(\Omega)} \mathrm{d} t=\frac{1}{2} \int_{Q_{\infty}} z^{2}\left(\partial_{1} \Phi+\partial_{2} \Phi\right)-\frac{1}{2} \int_{\Sigma_{\infty}} z^{2}\left(n_{1}+n_{2}\right) \Phi .
$$

Since $z$ belongs to $H^{1+\varepsilon, 1 / 2+\varepsilon / 2}\left(Q_{\infty}\right)$, from [9], Theorem B.3, it follows that $z^{2}$ belongs to $H^{2 \varepsilon, \varepsilon}\left(Q_{\infty}\right)$ and that

$$
\left\|z^{2}\right\|_{H^{2 \varepsilon, \varepsilon}\left(Q_{\infty}\right)} \leq C\|z\|_{H^{1+\varepsilon, 1 / 2+\varepsilon / 2}\left(Q_{\infty}\right)}^{2}
$$

Moreover, $z^{2} \in L^{2}\left(0, \infty ; H^{2 \varepsilon}(\Omega)\right) \subset L^{2}\left(0, \infty ; H^{\varepsilon}(\Omega)\right)$, and for $i=1,2$, we have

$$
\begin{aligned}
\left|\int_{Q_{\infty}} z^{2} \partial_{i} \Phi\right| & \leq C\left\|z^{2}\right\|_{L^{2}\left(0, \infty ; H^{\varepsilon}(\Omega)\right)}\left\|\partial_{i} \Phi\right\|_{L^{2}\left(0, \infty ; \mathcal{H}^{-\varepsilon}(\Omega)\right)} \\
& \leq C\left\|z^{2}\right\|_{H^{2 \varepsilon, \varepsilon}\left(Q_{\infty}\right)}\|\Phi\|_{L^{2}\left(0, \infty ; \mathcal{H}^{1-\varepsilon}(\Omega)\right)} .
\end{aligned}
$$

Thanks to estimate (5.3) we obtain

$$
\left|\int_{Q_{\infty}} z^{2} \partial_{i} \Phi\right| \leq C\|z\|_{H^{1+\varepsilon, 1 / 2+\varepsilon / 2}\left(Q_{\infty}\right)}^{2}\|\Phi\|_{L^{2}\left(0, \infty ; \mathcal{H}^{1-\varepsilon}(\Omega)\right)}
$$

Let us consider the second term. From the trace theorem in [15], Theorem 2.1, p. 10, since $\varepsilon>1 / 4$ we have $\left.z^{2}\right|_{\Sigma_{\infty}} \in H^{2 \varepsilon-1 / 2, \varepsilon-1 / 4}\left(\Sigma_{\infty}\right)$. Moreover, $\left.\Phi\right|_{\Sigma_{\infty}}$ belongs to $L^{2}\left(0, \infty ; H^{1 / 2-\varepsilon}(\Gamma)\right)$, and therefore $\left.\Phi\right|_{\Sigma_{\infty}} \in L^{2}\left(\Sigma_{\infty}\right)$ 
since $\varepsilon<1 / 2$. We finally obtain

$$
\begin{aligned}
\left|\int_{\Sigma_{\infty}} z^{2}\left(n_{1}+n_{2}\right) \Phi\right| & \leq C\left\|z^{2}\right\|_{H^{2 \varepsilon-1 / 2, \varepsilon-1 / 4}\left(\Sigma_{\infty}\right)}\|\Phi\|_{L^{2}\left(0, \infty ; \mathcal{H}^{1-\varepsilon}(\Omega)\right)} \\
& \leq C\left\|z^{2}\right\|_{H^{2 \varepsilon, \varepsilon}\left(Q_{\infty}\right)}\|\Phi\|_{L^{2}\left(0, \infty ; \mathcal{H}^{1-\varepsilon}(\Omega)\right)} \\
& \leq C\|z\|_{H^{1+\varepsilon, 1 / 2+\varepsilon / 2}\left(Q_{\infty}\right)}^{2}\|\Phi\|_{L^{2}\left(0, \infty ; \mathcal{H}^{1-\varepsilon}(\Omega)\right)}
\end{aligned}
$$

and the proof is complete.

Lemma 5.2. Let $\varepsilon$ belong to the interval $] 1 / 4,1 / 2[$. The mapping $F$ is locally Lipschitz continuous from $H^{1+\varepsilon, 1 / 2+\varepsilon / 2}\left(Q_{\infty}\right)$ into $L^{2}\left(0, \infty ; \mathcal{H}^{-1+\varepsilon}(\Omega)\right)$. More precisely, we have

$$
\left\|F\left(z_{1}\right)-F\left(z_{2}\right)\right\|_{L^{2}\left(0, \infty ; \mathcal{H}^{-1+\varepsilon}(\Omega)\right)} \leq C_{2}\left(\left\|z_{1}\right\|_{H^{1+\varepsilon, 1 / 2+\varepsilon / 2}\left(Q_{\infty}\right)}+\left\|z_{2}\right\|_{H^{1+\varepsilon, 1 / 2+\varepsilon / 2}\left(Q_{\infty}\right)}\right)\left\|z_{1}-z_{2}\right\|_{H^{1+\varepsilon, 1 / 2+\varepsilon / 2}\left(Q_{\infty}\right)},
$$

where the constant $C_{2}$ is the same constant as in Lemma 5.1.

Proof. We first write

$$
\int_{0}^{\infty}\left\langle F\left(z_{1}\right)-F\left(z_{2}\right), \Phi\right\rangle_{\mathcal{H}^{-1+\varepsilon}(\Omega), \mathcal{H}^{1-\varepsilon}(\Omega)}=\frac{1}{2} \int_{Q_{\infty}}\left(z_{1}^{2}-z_{2}^{2}\right)\left(\partial_{1} \Phi+\partial_{2} \Phi\right)-\frac{1}{2} \int_{\Sigma_{\infty}}\left(z_{1}^{2}-z_{2}^{2}\right) \Phi\left(n_{1}+n_{2}\right) .
$$

As in the proof of Lemma 5.1, for $i=1,2$ we obtain

$$
\begin{aligned}
\left|\int_{Q_{\infty}}\left(z_{1}^{2}-z_{2}^{2}\right) \partial_{i} \Phi\right| & \leq C\left\|\left(z_{1}^{2}-z_{2}^{2}\right)\right\|_{L^{2}\left(0, \infty ; H^{\varepsilon}(\Omega)\right)}\left\|\partial_{i} \Phi\right\|_{L^{2}\left(0, \infty ; \mathcal{H}^{-\varepsilon}(\Omega)\right)} \\
& \leq C\left\|\left(z_{1}-z_{2}\right)\left(z_{1}+z_{2}\right)\right\|_{H^{2 \varepsilon, \varepsilon}\left(Q_{\infty}\right)}\|\Phi\|_{L^{2}\left(0, \infty ; \mathcal{H}^{1-\varepsilon}(\Omega)\right)} .
\end{aligned}
$$

Then, from [9], Theorem B.3, it follows that

$$
\left|\int_{Q_{\infty}}\left(z_{1}^{2}-z_{2}^{2}\right) \partial_{i} \Phi\right| \leq C\left\|z_{1}-z_{2}\right\|_{H^{1+\varepsilon, 1 / 2+\varepsilon / 2}\left(Q_{\infty}\right)}\left\|z_{1}+z_{2}\right\|_{H^{1+\varepsilon, 1 / 2+\varepsilon / 2}\left(Q_{\infty}\right)}\|\Phi\|_{L^{2}\left(0, \infty ; \mathcal{H}^{1-\varepsilon}(\Omega)\right)} .
$$

Let us consider the second term. As in the proof of Lemma 5.1, we have

$$
\begin{aligned}
\left|\int_{\Sigma_{\infty}}\left(z_{1}^{2}-z_{2}^{2}\right) \Phi\left(n_{1}+n_{2}\right)\right| & \leq C\left\|z_{1}^{2}-z_{2}^{2}\right\|_{H^{2 \varepsilon-1 / 2, \varepsilon-1 / 4}\left(\Sigma_{\infty}\right)}\|\Phi\|_{L^{2}\left(0, \infty ; \mathcal{H}^{1-\varepsilon}(\Omega)\right)} \\
& \leq C\left\|\left(z_{1}-z_{2}\right)\left(z_{1}+z_{2}\right)\right\|_{H^{2 \varepsilon, \varepsilon}\left(Q_{\infty}\right)}\|\Phi\|_{L^{2}\left(0, \infty ; \mathcal{H}^{1-\varepsilon}(\Omega)\right)} \\
& \leq C\left\|z_{1}-z_{2}\right\|_{H^{1+\varepsilon, 1 / 2+\varepsilon / 2}\left(Q_{\infty}\right)}\left\|z_{1}+z_{2}\right\|_{H^{1+\varepsilon, 1 / 2+\varepsilon / 2}\left(Q_{\infty}\right)}\|\Phi\|_{L^{2}\left(0, \infty ; \mathcal{H}^{1-\varepsilon}(\Omega)\right)}
\end{aligned}
$$

and the proof is complete.

Lemma 5.3. Let $\varepsilon$ belong to $\left[0,1 / 2\left[\right.\right.$. If $h \in H^{\varepsilon}(\Omega)$, then

$$
\left|A_{\Pi}^{-*} h\right|_{H^{2+\varepsilon}(\Omega)} \leq C_{3}|h|_{H^{\varepsilon}(\Omega)} .
$$

Proof. We want to show that, for all $h \in H^{\varepsilon}(\Omega)$, the solution $y$ to $A_{\Pi}^{*} y=h$ belongs to $H^{2+\varepsilon}(\Omega)$. Since $h$ belongs to $L^{2}(\Omega)$, due to Lemma $3.3 y$ belongs to $D\left(A_{\Pi}^{*}\right)=D\left(A^{*}\right)$. Moreover, $y$ is solution to

$$
\left(A^{*}-\lambda_{0} I\right) y=h+\Pi B M^{2} B^{*} y-\lambda_{0} y .
$$


As $y$ belongs to $D\left(A^{*}\right)$, due to Proposition $3.1, M^{2} B^{*} y$ belongs to $H^{3 / 2}(\Gamma)$. For $1 / 2<\theta \leq 2$, since $B \in$ $\mathcal{L}\left(L^{2}(\Gamma), \mathcal{H}^{-\theta}(\Omega)\right)$ and $\varepsilon<1$, we have

$$
\left|B M^{2} B^{*} y\right|_{\mathcal{H}^{-1+\varepsilon / 2}(\Omega)} \leq C\left|M^{2} B^{*} y\right|_{L^{2}(\Gamma)} .
$$

Since $\Pi \in \mathcal{L}\left(L^{2}(\Omega), D\left(A^{*}\right)\right)$ and $\Pi \in \mathcal{L}\left(D\left(A^{*}\right)^{\prime}, L^{2}(\Omega)\right)$ (see Lem. 3.2), by interpolation we have

$$
\Pi \in \mathcal{L}\left(\mathcal{H}^{-2+\varepsilon}(\Omega), \mathcal{H}^{\varepsilon}(\Omega)\right)
$$

We finally obtain

$$
\left|\Pi B M^{2} B^{*} y\right|_{H^{\varepsilon}(\Omega)} \leq C\left|M^{2} B^{*} y\right|_{L^{2}(\Gamma)} .
$$

We have to solve

$$
\left(A^{*}-\lambda_{0} I\right) y=h_{1}
$$

with $h_{1}=h+\Pi B M^{2} B^{*} y-\lambda_{0} y \in H^{\varepsilon}(\Omega)$. The proof follows from regularity results for elliptic equations.

Lemma 5.4. Assume that $1 / 4<\varepsilon<1 / 2$. For all $z \in H^{1+\varepsilon, 1 / 2+\varepsilon / 2}\left(Q_{\infty}\right)$, we have

$$
\|G(z)\|_{L^{2}\left(\Sigma_{\infty}\right)} \leq C_{4}\|z\|_{H^{1+\varepsilon, 1 / 2+\varepsilon / 2}\left(Q_{\infty}\right)}^{2} .
$$

(The constant $C_{4}$ depends on $\varepsilon$.)

Proof. Let us recall that $G(z)=M B^{*} A_{\Pi}^{-*} \Pi F(z)$. Due to Lemma $5.1, F(z)$ belongs to $L^{2}\left(0, \infty ; \mathcal{H}^{-1+\varepsilon}(\Omega)\right)$ and

$$
\|F(z)\|_{L^{2}\left(0, \infty ; \mathcal{H}^{-1+\varepsilon}(\Omega)\right)} \leq C_{2}\|z\|_{H^{1+\varepsilon, 1 / 2+\varepsilon / 2}\left(Q_{\infty}\right)}^{2} .
$$

Moreover, using Lemma 3.2, by interpolation, $\Pi \in \mathcal{L}\left(\mathcal{H}^{-1+\varepsilon}(\Omega), \mathcal{H}^{1+\varepsilon}(\Omega)\right)$. Thus,

$$
\|\Pi F(z)\|_{L^{2}\left(0, \infty ; \mathcal{H}^{1+\varepsilon}(\Omega)\right)} \leq C\|z\|_{H^{1+\varepsilon, 1 / 2+\varepsilon / 2}\left(Q_{\infty}\right)}^{2} .
$$

Then, thanks to Lemma 5.3, since $\mathcal{H}^{1+\varepsilon}(\Omega) \subset H^{\varepsilon}(\Omega)$, we have

$$
\left\|A_{\Pi}^{-*} \Pi F(z)\right\|_{L^{2}\left(0, \infty ; H^{2+\varepsilon}(\Omega)\right)} \leq C\|z\|_{H^{1+\varepsilon, 1 / 2+\varepsilon / 2}\left(Q_{\infty}\right)}^{2} .
$$

Finally, with Proposition 3.1 we obtain

$$
\left\|M B^{*} A_{\Pi}^{-*} \Pi F(z)\right\|_{L^{2}\left(0, \infty ; H^{1 / 2+\varepsilon}(\Gamma)\right)} \leq C\|z\|_{H^{1+\varepsilon, 1 / 2+\varepsilon / 2}\left(Q_{\infty}\right)}^{2}
$$

and the proof is complete.

Lemma 5.5. Assume that $1 / 4<\varepsilon<1 / 2$. For all $z_{1}$ and $z_{2}$ in $H^{1+\varepsilon, 1 / 2+\varepsilon / 2}\left(Q_{\infty}\right)$, we have

$$
\left\|G\left(z_{1}\right)-G\left(z_{2}\right)\right\|_{L^{2}\left(\Sigma_{\infty}\right)} \leq C_{4}\left(\left\|z_{1}\right\|_{H^{1+\varepsilon, 1 / 2+\varepsilon / 2}\left(Q_{\infty}\right)}+\left\|z_{2}\right\|_{H^{1+\varepsilon, 1 / 2+\varepsilon / 2}\left(Q_{\infty}\right)}\right)\left\|z_{1}-z_{2}\right\|_{H^{1+\varepsilon, 1 / 2+\varepsilon / 2}\left(Q_{\infty}\right)},
$$

where the constant $C_{4}$ is the same constant as in Lemma 5.4. 
Proof. In Lemma 5.2, we have proved that $F$ is locally Lipschitz continuous from $H^{1+\varepsilon, 1 / 2+\varepsilon / 2}\left(Q_{\infty}\right)$ into $L^{2}\left(0, \infty ; \mathcal{H}^{-1+\varepsilon}(\Omega)\right)$. Then, following the proof of Lemma 5.4, the result follows easily.

\subsection{Proof of Theorem $\mathbf{5 . 1}$}

Proof. We set

$$
\mu_{0}=\frac{1}{4 C_{1}\left(C_{2}+C_{4}\right)} \quad \text { and } \quad \eta(\mu)=\frac{3}{4 C_{1}} \mu
$$

where the constants $C_{1}, C_{2}$ and $C_{4}$ are defined respectively in Theorem 4.1, Lemmas 5.1 and 5.4. For all $z \in H^{1+\varepsilon, 1 / 2+\varepsilon / 2}\left(Q_{\infty}\right)$, we denote by $y_{z}$ the solution to the equation

$$
y^{\prime}=A_{\Pi} y+F(z)+B M^{2} B^{*} A_{\Pi}^{-*} \Pi F(z), \quad y(0)=y_{0} .
$$

We are going to prove that the mapping $\mathbf{M}: z \mapsto y_{z}$ is a contraction in $D_{\mu}$.

(i) Let us take $z$ in $D_{\mu}$. From Lemma $5.1, F(z)$ belongs to $L^{2}\left(0, \infty ; \mathcal{H}^{-1+\varepsilon}(\Omega)\right)$. Moreover, from Lemma 5.4 and Theorem 4.1, $G(z)$ belongs to $L^{2}\left(\Sigma_{\infty}\right)$ and

$$
\left\|y_{z}\right\|_{H^{1+\varepsilon, 1 / 2+\varepsilon / 2}\left(Q_{\infty}\right)} \leq C_{1}\left(\left|y_{0}\right|_{H^{\varepsilon}(\Omega)}+\|G(z)\|_{L^{2}\left(\Sigma_{\infty}\right)}+\|F(z)\|_{L^{2}\left(0, \infty ; \mathcal{H}^{-1+\varepsilon}(\Omega)\right)}\right) .
$$

Then, using Lemmas 5.1 and 5.4, we have

$$
\begin{aligned}
\left\|y_{z}\right\|_{H^{1+\varepsilon, 1 / 2+\varepsilon / 2}\left(Q_{\infty}\right)} & \leq C_{1}\left(\left|y_{0}\right|_{H^{\varepsilon}(\Omega)}+C_{4}\|z\|_{H^{1+\varepsilon, 1 / 2+\varepsilon / 2}\left(Q_{\infty}\right)}^{2}+C_{2}\|z\|_{H^{1+\varepsilon, 1 / 2+\varepsilon / 2}\left(Q_{\infty}\right)}^{2}\right) \\
& \leq C_{1}\left(\frac{3}{4 C_{1}} \mu+\left(C_{2}+C_{4}\right)\|z\|_{H^{1+\varepsilon, 1 / 2+\varepsilon / 2}\left(Q_{\infty}\right)}^{2}\right) \\
& \leq \frac{3}{4} \mu+C_{1}\left(C_{2}+C_{4}\right) \mu^{2}<\mu \text { if } \mu<\mu_{0} .
\end{aligned}
$$

Thus $\mathbf{M}$ is a mapping from $D_{\mu}$ into itself.

(ii) Let us consider $z_{1}$ and $z_{2}$ in $D_{\mu}$. From Theorem 4.1, it follows that

$$
\left\|y_{z_{1}}-y_{z_{2}}\right\|_{H^{1+\varepsilon, 1 / 2+\varepsilon / 2}\left(Q_{\infty}\right)} \leq C_{1}\left(\left\|G\left(z_{1}\right)-G\left(z_{2}\right)\right\|_{L^{2}\left(\Sigma_{\infty}\right)}+\left\|F\left(z_{1}\right)-F\left(z_{2}\right)\right\|_{L^{2}\left(0, \infty ; \mathcal{H}^{-1+\varepsilon}(\Omega)\right)}\right) .
$$

If $\mu<\mu_{0}$, using Lemmas 5.2 and 5.5 , we have

$$
\begin{aligned}
\left\|y_{z_{1}}-y_{z_{2}}\right\|_{H^{1+\varepsilon, 1 / 2+\varepsilon / 2}\left(Q_{\infty}\right)} & \leq C_{1}\left(C_{2}+C_{4}\right)\left(\left\|z_{1}\right\|_{H^{1+\varepsilon, 1 / 2+\varepsilon / 2}\left(Q_{\infty}\right)}+\left\|z_{2}\right\|_{H^{1+\varepsilon, 1 / 2+\varepsilon / 2}\left(Q_{\infty}\right)}\right)\left\|z_{1}-z_{2}\right\|_{H^{1+\varepsilon, 1 / 2+\varepsilon / 2}\left(Q_{\infty}\right)} \\
& \leq 2 C_{1}\left(C_{2}+C_{4}\right) \mu\left\|z_{1}-z_{2}\right\|_{H^{1+\varepsilon, 1 / 2+\varepsilon / 2}\left(Q_{\infty}\right)} \leq \frac{1}{2}\left\|z_{1}-z_{2}\right\|_{H^{1+\varepsilon, 1 / 2+\varepsilon / 2}\left(Q_{\infty}\right)} .
\end{aligned}
$$

The mapping $\mathbf{M}$ is a contraction in $D_{\mu}$ and system (5.1) admits a unique solution in the set $D_{\mu}$.

\section{Stabilization with a given decay Rate}

In this section, we explain how to adapt the results of Section 5 to obtain a prescribed exponential decay rate $-\alpha<0$. We briefly recall the method used in [16]. For that, we set

$$
\hat{y}=\mathrm{e}^{\alpha t} y, \quad \hat{u}=\mathrm{e}^{\alpha t} u .
$$

If

$$
y^{\prime}=A y+F(y)+B M u, \quad y(0)=y_{0},
$$


then $\hat{y}$ is solution to the system

$$
\hat{y}^{\prime}=(A+\alpha I) \hat{y}+\mathrm{e}^{-\alpha t} F(\hat{y})+B M \hat{u}, \quad \hat{y}(0)=y_{0} .
$$

Let us set $A_{\alpha}=A+\alpha I$, and let $\Pi_{\alpha} \in \mathcal{L}\left(L^{2}(\Omega)\right)$ be the solution to the algebraic Riccati equation

$$
\begin{aligned}
& \Pi_{\alpha}=\Pi_{\alpha}^{*} \in \mathcal{L}\left(L^{2}(\Omega)\right), \quad \Pi_{\alpha} \geq 0, \\
& \left|\Pi_{\alpha} y\right|_{D\left(A^{*}\right)} \leq|y|_{L^{2}(\Omega)} \quad \text { for all } y \in L^{2}(\Omega), \\
& \Pi_{\alpha} A_{\alpha}+A_{\alpha}^{*} \Pi_{\alpha}-\Pi_{\alpha} B M^{2} B^{*} \Pi_{\alpha}+I=0 .
\end{aligned}
$$

Now, we consider the nonlinear law

$$
\hat{u}=-M B^{*} \Pi_{\alpha} \hat{y}+M B^{*} A_{\alpha, \Pi_{\alpha}}^{-*} \Pi_{\alpha} \mathrm{e}^{-\alpha t} F(\hat{y}),
$$

where

$$
D\left(A_{\alpha, \Pi_{\alpha}}\right)=\left\{y \in H^{2}(\Omega) \mid \nu \frac{\partial y}{\partial n}+M^{2} B^{*} \Pi_{\alpha} y=0 \text { on } \Gamma\right\}, \quad A_{\alpha, \Pi_{\alpha}} y=A_{\alpha} y-B M^{2} B^{*} \Pi_{\alpha} y
$$

for all $y \in D\left(A_{\alpha, \Pi_{\alpha}}\right)=D\left(A_{\Pi_{\alpha}}\right)$. Then, we have to study the following system

$$
\hat{y}^{\prime}=A_{\alpha, \Pi_{\alpha}} \hat{y}+B M^{2} B^{*} A_{\alpha, \Pi_{\alpha}}^{-*} \Pi_{\alpha} \mathrm{e}^{-\alpha t} F(\hat{y})+\mathrm{e}^{-\alpha t} F(\hat{y}), \quad \hat{y}(0)=y_{0} .
$$

We can remark that $A_{\alpha, \Pi_{\alpha}} y=A_{\Pi_{\alpha}} y+\alpha y$.

Theorem 6.1. For all $1 / 4<\varepsilon<1 / 2$, there exist $\mu_{0}>0$ and a nondecreasing function $\eta$ from $\mathbb{R}^{+}$into itself, such that if $\mu \in\left(0, \mu_{0}\right)$ and $\left|y_{0}\right|_{H^{\varepsilon}(\Omega)} \leq \eta(\mu)$, then equation (6.1) admits a unique solution in the set

$$
D_{\mu, \alpha}=\left\{y \in H^{1+\varepsilon, 1 / 2+\varepsilon / 2}\left(Q_{\infty}\right) \mid\left\|\mathrm{e}^{\alpha(\cdot)} y\right\|_{H^{1+\varepsilon, 1 / 2+\varepsilon / 2}\left(Q_{\infty}\right)} \leq \mu\right\} .
$$

Proof. We consider equation (6.3) and we want to show that this equation admits a unique solution $\hat{y} \in D_{\mu, 0}$. We substitute $F(y)$ by $\mathrm{e}^{-\alpha t} F(\hat{y}), A$ by $A+\alpha I$ and $\Pi$ by $\Pi_{\alpha}$, and the proof follows the lines of Theorem 5.1 .

\section{Dirichlet Boundary CONTROL}

In this section, we are going to study the case of a Dirichlet boundary control. Let $w$ be a solution to the stationary Burgers equation in $\Omega$ with Dirichlet boundary conditions

$$
-\nu \Delta w+w\left(\partial_{1} w+\partial_{2} w\right)=f \quad \text { in } \Omega, \quad w=g \quad \text { on } \Gamma .
$$

The purpose of this part is to determine a boundary control $u$, in feedback form, localized in a part of the boundary $\Gamma$, so that the corresponding control system

$$
\left\{\begin{array}{l}
\partial_{t} z-\nu \Delta z+z\left(\partial_{1} z+\partial_{2} z\right)=f \quad \text { in } \Omega \times(0, \infty) \\
z=g+M u \quad \text { on } \Sigma_{\infty}, \quad z(0)=w+y_{0} \quad \text { in } \Omega
\end{array}\right.
$$

is exponentially stable with a prescribed decay rate, for initial values $y_{0}$ small enough in $L^{2}(\Omega)$ (or more generally in $H^{\varepsilon}(\Omega)$ with $0 \leq \varepsilon<1 / 2$ ). The operator $M$ is defined as in Section 2. Setting $y=z-w$, the function $y$ obeys

$$
\left\{\begin{array}{l}
\partial_{t} y-\nu \Delta y+y\left(\partial_{1} w+\partial_{2} w\right)+w\left(\partial_{1} y+\partial_{2} y\right)+y\left(\partial_{1} y+\partial_{2} y\right)=0 \quad \text { in } \Omega \times(0, \infty) \\
y=M u \text { on } \Sigma_{\infty}, \quad y(0)=y_{0} \quad \text { in } \Omega
\end{array}\right.
$$


We denote by $(A, D(A))$ and $\left(A^{*}, D\left(A^{*}\right)\right)$ the unbounded operators in $L^{2}(\Omega)$ defined by

$$
\begin{array}{ll}
D(A)=H^{2}(\Omega) \cap H_{0}^{1}(\Omega), & A y=\nu \Delta y-y\left(\partial_{1} w+\partial_{2} w\right)-w\left(\partial_{1} y+\partial_{2} y\right), \\
D\left(A^{*}\right)=H^{2}(\Omega) \cap H_{0}^{1}(\Omega), & A^{*} y=\nu \Delta y+w\left(\partial_{1} y+\partial_{2} y\right) .
\end{array}
$$

There exists $\lambda_{0}>0$ an element in the resolvent set of $A$ for which the coercivity conditions stated in (2.2) in the case of Neumann boundary conditions are still true for the above operators. We denote by $D \in \mathcal{L}\left(L^{2}(\Gamma), L^{2}(\Omega)\right)$ the operator defined by $D u=y$, where $y$ is the unique solution in $H^{1 / 2}(\Omega)$ to the equation

$$
\lambda_{0} y-\nu \Delta y+w\left(\partial_{1} y+\partial_{2} y\right)+y\left(\partial_{1} w+\partial_{2} w\right)=0 \quad \text { in } \Omega, \quad y=u \quad \text { on } \Gamma .
$$

The nonlinear term $-y\left(\partial_{1} y+\partial_{2} y\right)$ is rewritten as an element $F(y)$ in $\left(D\left(A^{*}\right)\right)^{\prime}$ as follows

$$
\langle F(y), \Phi\rangle_{\left(D\left(A^{*}\right)\right)^{\prime}, D\left(A^{*}\right)}=\frac{1}{2} \int_{\Omega} y^{2}\left(\partial_{1} \Phi+\partial_{2} \Phi\right) \quad \text { for all } \quad \Phi \in D\left(A^{*}\right) .
$$

Let us observe that $F(y)$ is well defined in $\left(D\left(A^{*}\right)\right)^{\prime}$ for all $y \in H^{1}(\Omega)$. Setting $B=\left(\lambda_{0} I-A\right) D$, equation (7.3) may be rewritten in the form

$$
y^{\prime}=A y+B M u+F(y) \quad \text { in }(0, \infty), \quad y(0)=y_{0} .
$$

As for a Neumann boundary control, we want to study the following nonlinear feedback law

$$
u=-M B^{*} \Pi y+M B^{*} A_{\Pi}^{-*} \Pi F(y),
$$

where $\Pi$ is the solution to the algebraic equation (2.5) and $A_{\Pi}$ is defined in Section 2, except that now $A$ is defined by (7.4) and $B=\left(\lambda_{0} I-A\right) D$. We indicate in the following the results corresponding to a Dirichlet boundary control, and we give the proofs only when they differ from the case of a Neumann boundary control.

The stabilizability of the pair $(A, B M)$ follows from null controllability results for advection-diffusion equations with homogeneous Dirichlet boundary conditions and with a distributed control (see e.g. [7] and the references therein).

\subsection{Properties of some operators}

The analogues of Theorem 3.1, Lemma 3.1, and Proposition 3.1 are stated below. The statement of Lemma 3.3 can be rewritten word for word in the case of Dirichlet boundary conditions. More precisely, we have

$$
D\left(A_{\Pi}\right)=\left\{y \in H^{2}(\Omega) \mid y+M^{2} B^{*} \Pi y=0 \text { on } \Gamma\right\} \quad \text { and } \quad D\left(A_{\Pi}^{*}\right)=D(A) .
$$

Theorem 7.1. The unbounded operator $\left(A-\lambda_{0} I\right)$ (respectively $\left.\left(A^{*}-\lambda_{0} I\right)\right)$ with domain $D\left(A-\lambda_{0} I\right)=D(A)$ (respectively $D\left(A^{*}-\lambda_{0} I\right)=D\left(A^{*}\right)$ ) is the infinitesimal generator of a bounded analytic semigroup exponentially stable on $L^{2}(\Omega)$. Moreover, we have

$$
D\left(\left(\lambda_{0} I-A\right)^{\alpha}\right)=D\left(\left(\lambda_{0} I-A^{*}\right)^{\alpha}\right)=\left\{\begin{array}{l}
H^{2 \alpha}(\Omega) \quad \text { if } \alpha \in[0,1 / 4[ \\
\left.\left.\left\{z \in H^{2 \alpha}(\Omega) \mid z=0 \text { on } \Gamma\right\} \quad \text { if } \alpha \in\right] 1 / 4,1\right] .
\end{array}\right.
$$

Lemma 7.1. The operator D satisfies

$$
|D u|_{H^{s+1 / 2}(\Omega)} \leq C(s)|u|_{H^{s}(\Gamma)} \quad \text { for all } 0 \leq s \leq 2 .
$$


The operator $D^{*}$, the adjoint operator of $D \in \mathcal{L}\left(L^{2}(\Gamma), L^{2}(\Omega)\right)$, is defined by

$$
D^{*} g=-\nu \frac{\partial z}{\partial n}
$$

where $z$ is the solution of

$$
\lambda_{0} z-\nu \Delta z-w\left(\partial_{1} z+\partial_{2} z\right)=g \quad \text { in } \Omega, \quad z=0 \quad \text { on } \Gamma .
$$

Proposition 7.1. For all $\Phi \in D\left(A^{*}\right), B^{*} \Phi$ is defined by

$$
B^{*} \Phi=D^{*}\left(\lambda_{0} I-A^{*}\right) \Phi=-\nu \frac{\partial \Phi}{\partial n},
$$

and

$$
\left|M B^{*} \Phi\right|_{H^{s-3 / 2}(\Gamma)} \leq C|\Phi|_{H^{s}(\Omega)} \quad \text { if } 3 / 2<s<5 / 2
$$

\subsection{Nonhomogeneous equations}

In this subsection and the following one, the constants $C_{1}, \ldots, C_{4}$ are not necessarily the same as in Section 5 . We first state optimal regularity results for the solution to the equation

$$
y^{\prime}=\left(A-\lambda_{0} I\right) y+B M h, \quad y(0)=0 .
$$

Lemma 7.2. Assume that $h \in H^{s, s / 2}\left(\Sigma_{\infty}\right)$ with $0 \leq s \leq 3 / 2, s \neq 1$, and that $h(\cdot, 0)=0$ on $\Gamma$ if $s>1$. Then, the solution of equation (7.8) obeys

$$
\|y\|_{H^{s+1 / 2, s / 2+1 / 4}\left(Q_{\infty}\right)} \leq C\|h\|_{H^{s, s / 2}\left(\Sigma_{\infty}\right)} .
$$

Proof. The proof relies on [15], Chapter 4, Part 13.

We now study the equation

$$
y^{\prime}=A_{\Pi} y+f+B M g, \quad y(0)=y_{0} .
$$

Theorem 7.2. If $f \in L^{2}\left(0, \infty ; \mathcal{H}^{-1+\varepsilon}(\Omega)\right), g \in H^{1 / 2+\varepsilon, 1 / 4+\varepsilon / 2}\left(\Sigma_{\infty}\right), y_{0} \in H^{\varepsilon}(\Omega)$ with $0 \leq \varepsilon<1 / 2$, then equation (7.9) admits a unique solution in $L^{2}\left(0, \infty ; L^{2}(\Omega)\right) \cap H^{1}\left(0, \infty ;\left(D\left(A^{*}\right)\right)^{\prime}\right)$ which obeys

$$
\|y\|_{H^{1+\varepsilon, 1 / 2+\varepsilon / 2}\left(Q_{\infty}\right)} \leq C_{1}\left(\left|y_{0}\right|_{H^{\varepsilon}(\Omega)}+\|f\|_{L^{2}\left(0, \infty ; \mathcal{H}^{1-\varepsilon}(\Omega)\right)}+\|g\|_{H^{1 / 2+\varepsilon, 1 / 4+\varepsilon / 2}\left(\Sigma_{\infty}\right)}\right) .
$$

Proof. We use the same strategy as in the proof of Theorem 4.1. In particular $y_{1}$ and $y_{2}$ are defined in a similar way. First notice that the existence of $y$ in $L^{2}\left(Q_{\infty}\right)$ and the estimate of $y_{1}$ can be obtained as in the proof of Theorem 4.1. The only difference is in the estimate of $y_{2}$. Let us recall that $y_{2}$ is the solution to

$$
y_{2}^{\prime}=\left(A-\lambda_{0} I\right) y_{2}+B M h, \quad y_{2}(0)=0,
$$

with $h=g-M B^{*} \Pi y$. Since $y$ belongs to $L^{2}\left(Q_{\infty}\right)$, the function $h=g-M B^{*} \Pi y$ belongs to $L^{2}\left(0, \infty ; H^{1 / 2}(\Gamma)\right)$. With Lemma 7.2 we have

$$
\left\|y_{2}\right\|_{H^{1 / 2,1 / 4}\left(Q_{\infty}\right)} \leq C\|h\|_{L^{2}\left(\Sigma_{\infty}\right)} \leq C\left(\|g\|_{H^{1 / 2+\varepsilon, 1 / 4+\varepsilon / 2}\left(\Sigma_{\infty}\right)}+\|y\|_{L^{2}\left(Q_{\infty}\right)}\right) .
$$

Thus, with the estimate on $y_{1}$, it follows that $y$ belongs to $H^{1 / 2,1 / 4}\left(Q_{\infty}\right)$ and $h \in H^{1 / 2,1 / 4}\left(\Sigma_{\infty}\right)$. Still using Lemma 7.2 , the solution $y_{2}$ belongs to $H^{1,1 / 2}\left(Q_{\infty}\right)$, and

$$
\left\|y_{2}\right\|_{H^{1,1 / 2}\left(Q_{\infty}\right)} \leq C\|h\|_{H^{1 / 2,1 / 4}\left(\Sigma_{\infty}\right)} \leq C\left(\|g\|_{H^{1 / 2+\varepsilon, 1 / 4+\varepsilon / 2}\left(\Sigma_{\infty}\right)}+\|y\|_{H^{1 / 2,1 / 4}\left(Q_{\infty}\right)}\right)
$$


Therefore $y$ belongs to $H^{1,1 / 2}\left(Q_{\infty}\right)$. Since $M B^{*} \Pi \in \mathcal{L}\left(L^{2}(\Omega), L^{2}(\Gamma)\right)$, the term $M B^{*} \Pi y$ belongs to $H^{1 / 2}(0, \infty$; $\left.L^{2}(\Gamma)\right)$. Moreover, as in [16], Remark 4.4, $M B^{*} \Pi \in \mathcal{L}\left(H^{\varepsilon}(\Omega), H^{1 / 2+\varepsilon}(\Gamma)\right)$. Then, $M B^{*} \Pi y$ belongs to $L^{2}(0, \infty ;$ $\left.H^{1 / 2+\varepsilon}(\Gamma)\right)$. From these results, it follows that

$$
h \in H^{1 / 2+\varepsilon, 1 / 4+\varepsilon / 2}\left(\Sigma_{\infty}\right) .
$$

We still use Lemma 7.2 to prove that $y_{2}$ belongs to $H^{1+\varepsilon, 1 / 2+\varepsilon / 2}\left(Q_{\infty}\right)$, and we can conclude with the estimate on $y_{1}$.

\subsection{Stabilization of the two dimensional Burgers equation}

Consider the Burgers equation with the nonlinear feedback law

$$
y^{\prime}=A_{\Pi} y+B M^{2} B^{*} A_{\Pi}^{-*} \Pi F(y)+F(y), \quad y(0)=y_{0} .
$$

It has the same form as equation (5.1), but the operators $A_{\Pi}, B, \Pi$ are different.

Theorem 7.3. For all $0 \leq \varepsilon<1 / 2$, there exist $\mu_{0}>0$ and a nondecreasing function $\eta$ from $\mathbb{R}^{+}$into itself, such that if $\mu \in\left(0, \mu_{0}\right)$ and $\left|y_{0}\right|_{H^{\varepsilon}(\Omega)} \leq \eta(\mu)$, then equation (7.11) admits a unique solution in the set

$$
D_{\mu}=\left\{y \in H^{1+\varepsilon, 1 / 2+\varepsilon / 2}\left(Q_{\infty}\right) \mid\|y\|_{H^{1+\varepsilon, 1 / 2+\varepsilon / 2}\left(Q_{\infty}\right)} \leq \mu\right\} .
$$

As in Section 5, the proof of Theorem 7.3 follows from a fixed point theorem, and it relies on estimates for $F(z)$ and $G(z)=M B^{*} A_{\Pi}^{-*} \Pi F(z)$.

Lemma 7.3. If $0 \leq \varepsilon<1 / 2$ and $z$ belongs to $H^{1+\varepsilon, 1 / 2+\varepsilon / 2}\left(Q_{\infty}\right)$, then $F(z) \in L^{2}\left(0, \infty ; \mathcal{H}^{-1+\varepsilon}(\Omega)\right)$ and we have

$$
\|F(z)\|_{L^{2}\left(0, \infty ; \mathcal{H}^{-1+\varepsilon}(\Omega)\right)} \leq C_{2}\|z\|_{H^{1+\varepsilon, 1 / 2+\varepsilon / 2}\left(Q_{\infty}\right)}^{2} .
$$

Proof. The proof follows the lines of Lemma 5.1 and is easier because we only have to estimate the term

$$
\int_{0}^{\infty}\langle F(z), \Phi\rangle_{\mathcal{H}^{-1+\varepsilon}(\Omega), \mathcal{H}^{1-\varepsilon}(\Omega)}=\frac{1}{2} \int_{Q_{\infty}} z^{2}\left(\partial_{1} \Phi+\partial_{2} \Phi\right) .
$$

Similarly, we can prove the following lemma.

Lemma 7.4. $F$ is locally Lipschitz continuous from $H^{1+\varepsilon, 1 / 2+\varepsilon / 2}\left(Q_{\infty}\right)$ into $L^{2}\left(0, \infty ; \mathcal{H}^{-1+\varepsilon}(\Omega)\right)$ for all $0 \leq$ $\varepsilon<1 / 2$. More precisely, we have

$$
\left\|F\left(z_{1}\right)-F\left(z_{2}\right)\right\|_{L^{2}\left(0, \infty ; \mathcal{H}^{-1+\varepsilon}(\Omega)\right)} \leq C_{2}\left(\left\|z_{1}\right\|_{H^{1+\varepsilon, 1 / 2+\varepsilon / 2}\left(Q_{\infty}\right)}+\left\|z_{2}\right\|_{H^{1+\varepsilon, 1 / 2+\varepsilon / 2}\left(Q_{\infty}\right)}\right)\left\|z_{1}-z_{2}\right\|_{H^{1+\varepsilon, 1 / 2+\varepsilon / 2}\left(Q_{\infty}\right)}
$$

(and the constant $C_{2}$ depends on $\varepsilon$ ).

Lemma 7.5. If $0 \leq \varepsilon<1 / 2$ and if $h \in H^{\varepsilon}(\Omega)$, then

$$
\left|A_{\Pi}^{-*} h\right|_{H^{2+\varepsilon}(\Omega)} \leq C_{3}|h|_{H^{\varepsilon}(\Omega)} .
$$

Proof. In the case of Dirichlet boundary conditions, $B$ belongs to $\mathcal{L}\left(L^{2}(\Gamma), \mathcal{H}^{-\theta}(\Omega)\right)$ for $3 / 2<\theta \leq 2$. Since $\varepsilon<1 / 2$, we have

$$
\left|B M^{2} B^{*} y\right|_{\mathcal{H}^{-2+\varepsilon}(\Omega)} \leq C\left|M^{2} B^{*} y\right|_{L^{2}(\Gamma)} .
$$

Then, the proof is similar to the one of Lemma 5.3. 
Lemma 7.6. Assume that $0 \leq \varepsilon<1 / 2$. For all $z \in H^{1+\varepsilon, 1 / 2+\varepsilon / 2}\left(Q_{\infty}\right)$, we have

$$
\|G(z)\|_{H^{1 / 2+\varepsilon, 1 / 4+\varepsilon / 2}\left(\Sigma_{\infty}\right)} \leq C_{4}\|z\|_{H^{1+\varepsilon, 1 / 2+\varepsilon / 2}\left(Q_{\infty}\right)}^{2}
$$

Proof. The proof of estimate in $L^{2}\left(0, \infty ; H^{1 / 2+\varepsilon}(\Gamma)\right)$ is similar to the one of Lemma 5.4. We want to prove that

$$
\|G(z)\|_{H^{1 / 4+\varepsilon / 2}\left(0, \infty ; L^{2}(\Gamma)\right)} \leq C_{4}\|z\|_{H^{1+\varepsilon, 1 / 2+\varepsilon / 2}\left(Q_{\infty}\right)}^{2}
$$

Step 1. We first show

$$
\left\|z^{2}\right\|_{H^{1 / 4+\varepsilon / 2}\left(0, \infty ; H^{-1 / 2+\varepsilon}(\Omega)\right)} \leq C\|z\|_{H^{3 / 8+\varepsilon / 4}\left(0, \infty ; H^{1 / 4+\varepsilon / 2}(\Omega)\right)}^{2} .
$$

To prove this estimate we cannot apply the results in [9] because the exponent $-1 / 2+\varepsilon$ is negative. We are going to use the same method as in [10].

Since $z$ belongs to $H^{1+\varepsilon, 1 / 2+\varepsilon / 2}\left(Q_{\infty}\right)$, by interpolation $z$ belongs to $H^{\sigma}\left(0, \infty ; H^{s}(\Omega)\right)$ with $\sigma=3 / 8+\varepsilon / 4$ and $s=1 / 4+\varepsilon / 2$. We look for $\tilde{\sigma}$ and $\tilde{s}$ such that $z^{2} \in H^{\tilde{\sigma}}\left(0, \infty ; H^{\tilde{s}}(\Omega)\right)$. To obtain such a result, we use an extension operator (extending functions defined in $\Omega \times(0, \infty)$ to functions defined in $\mathbb{R}^{n+1}$ with $n=2$ ) and the estimate for the extended function is obtained via a Fourier transform. There exists an extension operator $P \in \mathcal{L}\left(H^{1}(\Omega), H^{1}\left(\mathbb{R}^{n}\right)\right)$ such that $\left.P z\right|_{\Omega}=z$ for all $z \in H^{1}\left(\mathbb{R}^{n}\right)$, and moreover $P$ is also continuous from $L^{2}(\Omega)$ into $L^{2}\left(\mathbb{R}^{n}\right)$. We extend functions defined in $(0, \infty)$ to functions defined in $\mathbb{R}$ by symmetry. We denote by $\tilde{P}$ the extension operator consisting in first applying $P$ and next the extension with respect to the time variable. Since $\sigma \in(0,1)$ and $s \in(0,1)$, we have

$$
\|\tilde{P} z\|_{H^{\sigma}\left(\mathbb{R} ; H^{s}\left(\mathbb{R}^{n}\right)\right)} \leq C\|z\|_{H^{\sigma}\left(0, \infty ; H^{s}(\Omega)\right)} .
$$

Let us show that if $z$ belongs to $H^{\sigma}\left(\mathbb{R} ; H^{s}\left(\mathbb{R}^{n}\right)\right)$, then $z^{2}$ belongs to $H^{\tilde{\sigma}}\left(\mathbb{R} ; H^{\tilde{s}}\left(\mathbb{R}^{n}\right)\right)$ for some $\tilde{\sigma}$ and $\tilde{s}$ that we are going to characterize. The Fourier transform of $z^{2}$ in $\mathbb{R}^{n+1}$ obeys

$$
\left(1+|\tau|^{2}\right)^{\tilde{\sigma} / 2}\left(1+|\zeta|^{2}\right)^{\tilde{s} / 2} \widehat{z^{2}}(\tau, \zeta)=\int_{\mathbb{R}^{n+1}} G(\tilde{\zeta}, \tilde{\eta}) z_{1}(\tilde{\eta}) z_{1}(\tilde{\zeta}-\tilde{\eta}) \mathrm{d} \tilde{\eta}
$$

with

$$
\begin{aligned}
& \tilde{\zeta}=(\tau, \zeta) \in \mathbb{R} \times \mathbb{R}^{n}, \quad \tilde{\eta}=(\lambda, \eta) \in \mathbb{R} \times \mathbb{R}^{n}, \\
& z_{1}(\tilde{\eta})=\left(1+|\lambda|^{2}\right)^{\sigma / 2}\left(1+|\eta|^{2}\right)^{s / 2} \widehat{z}(\tilde{\eta}), \quad G(\tilde{\zeta}, \tilde{\eta})=G_{1}(\tau, \lambda) G_{2}(\zeta, \eta),
\end{aligned}
$$

and

$$
\left\{\begin{array}{l}
G_{1}(\tau, \lambda)=\left(1+|\lambda|^{2}\right)^{-\sigma / 2}\left(1+|\tau-\lambda|^{2}\right)^{-\sigma / 2}\left(1+|\tau|^{2}\right)^{\tilde{\sigma} / 2} \\
G_{2}(\zeta, \eta)=\left(1+|\eta|^{2}\right)^{-s / 2}\left(1+|\zeta-\eta|^{2}\right)^{-s / 2}\left(1+|\zeta|^{2}\right)^{\tilde{s} / 2}
\end{array}\right.
$$

Since $z$ belongs to $H^{\sigma}\left(\mathbb{R} ; H^{s}\left(\mathbb{R}^{n}\right)\right)$, we clearly have $z_{1} \in L^{2}\left(\mathbb{R}^{n+1}\right)$. We follow the proofs of [10], Theorem 8.3.1, p. 189, and [9], Theorem B.3. We recall that if $T_{G}(f, h)$ is defined by

$$
T_{G}(f, h)(\tilde{\zeta})=\int_{\mathbb{R}^{n+1}} G(\tilde{\zeta}, \tilde{\eta}) f(\tilde{\zeta}-\tilde{\eta}) h(\tilde{\eta}) \mathrm{d} \tilde{\eta}
$$

for $f$ and $h \in C_{0}^{\infty}\left(\mathbb{R}^{n+1}\right)$, then

$$
\left\|T_{G}(f, h)\right\|_{L^{2}\left(\mathbb{R}^{n+1}\right)} \leq B\|f\|_{L^{2}\left(\mathbb{R}^{n+1}\right)}\|h\|_{L^{2}\left(\mathbb{R}^{n+1}\right)}
$$


when either $(a),(b)$ or $(c)$ holds
(a) $\int_{\mathbb{R}^{n+1}}|G(\tilde{\zeta}, \tilde{\eta})|^{2} \mathrm{~d} \tilde{\eta} \leq B^{2}$ for all $\tilde{\zeta}$,
(b) $\int_{\mathbb{R}^{n+1}}|G(\tilde{\zeta}, \tilde{\eta})|^{2} \mathrm{~d} \tilde{\zeta} \leq B^{2}$ for all $\tilde{\eta}$,
(c) $\int_{\mathbb{R}^{n+1}}|G(\tilde{\zeta}, \tilde{\zeta}-\tilde{\eta})|^{2} \mathrm{~d} \tilde{\zeta} \leq B^{2}$ for all $\tilde{\eta}$.

We first remark that

$$
\left(1+|\tau|^{2}\right)^{\tilde{\sigma} / 2}\left(1+|\zeta|^{2}\right)^{\tilde{s} / 2} \widehat{z^{2}}(\tau, \zeta)=T_{G}\left(z_{1}, z_{1}\right)(\tilde{\zeta})
$$

and we look for $\tilde{\sigma}$ and $\tilde{s}$ such that $T_{G}\left(z_{1}, z_{1}\right)$ belongs to $L^{2}\left(\mathbb{R}^{n+1}\right)$. Moreover, we clearly have

$$
\int_{\mathbb{R}^{n+1}}|G(\tilde{\zeta}, \tilde{\eta})|^{2} \mathrm{~d} \tilde{\eta}=\int_{\mathbb{R}}\left|G_{1}(\tau, \lambda)\right|^{2} \mathrm{~d} \lambda \int_{\mathbb{R}^{n}}\left|G_{2}(\zeta, \eta)\right|^{2} \mathrm{~d} \eta .
$$

Therefore it is sufficient to prove $(a),(b)$ or $(c)$ for $G_{1}$ and $G_{2}$. Following the proof of [10], Theorem 8.3.1, p. 189, these conditions are satisfied for

$$
\tilde{\sigma}=1 / 4+\varepsilon / 2 \quad \text { and } \quad \tilde{s}=-1 / 2+\varepsilon .
$$

From (7.13), it follows that $T_{G}\left(z_{1}, z_{1}\right) \in L^{2}\left(\mathbb{R}^{n+1}\right)$, that is to say $z^{2} \in H^{1 / 4+\varepsilon / 2}\left(\mathbb{R} ; H^{-1 / 2+\varepsilon}\left(\mathbb{R}^{n}\right)\right)$, and

$$
\begin{aligned}
\left\|z^{2}\right\|_{H^{1 / 4+\varepsilon / 2}\left(0, \infty ; H^{-1 / 2+\varepsilon}(\Omega)\right)} & \leq\left\|z^{2}\right\|_{H^{1 / 4+\varepsilon / 2}\left(\mathbb{R} ; H^{-1 / 2+\varepsilon}\left(\mathbb{R}^{n}\right)\right)} \leq C\|z\|_{H^{3 / 8+\varepsilon / 4}\left(\mathbb{R} ; H^{1 / 4+\varepsilon / 2}\left(\mathbb{R}^{n}\right)\right)}^{2} \\
& \leq C\|z\|_{H^{3 / 8+\varepsilon / 4}\left(0, \infty ; H^{1 / 4+\varepsilon / 2}(\Omega)\right)}^{2} .
\end{aligned}
$$

Step 2. Estimate of $F(z)$.

The operator $\partial_{i}$ belongs to $\mathcal{L}\left(H^{-1 / 2+\varepsilon}(\Omega), \mathcal{H}^{-3 / 2+\varepsilon}(\Omega)\right)$. Indeed, for all $\Phi \in \mathcal{H}^{3 / 2-\varepsilon}(\Omega)=H_{0}^{3 / 2-\varepsilon}(\Omega)$ and all $y \in H^{-1 / 2+\varepsilon}(\Omega)$ we have

$$
\begin{aligned}
\left|\left\langle\partial_{i} y, \Phi\right\rangle_{\mathcal{H}^{-3 / 2+\varepsilon}(\Omega), \mathcal{H}^{3 / 2-\varepsilon}(\Omega)}\right| & =\left|-\left\langle y, \partial_{i} \Phi\right\rangle_{H^{-1 / 2+\varepsilon}(\Omega), H^{1 / 2-\varepsilon}(\Omega)}\right| \\
& \leq|y|_{H^{-1 / 2+\varepsilon}(\Omega)}\left|\partial_{i} \Phi\right|_{H^{1 / 2-\varepsilon}(\Omega)} \\
& \leq C|y|_{H^{-1 / 2+\varepsilon}(\Omega)}|\Phi|_{\mathcal{H}^{3 / 2-\varepsilon}(\Omega)} .
\end{aligned}
$$

It follows that

$$
\|F(z)\|_{H^{1 / 4+\varepsilon / 2}\left(0, \infty ; \mathcal{H}^{-3 / 2+\varepsilon}(\Omega)\right)} \leq C\|z\|_{H^{1+\varepsilon, 1 / 2+\varepsilon / 2}\left(Q_{\infty}\right)}^{2} .
$$

Step 3. Estimate of $G(z)$.

Since $\Pi \in \mathcal{L}\left(L^{2}(\Omega), D\left(A^{*}\right)\right)$ and $\Pi \in \mathcal{L}\left(\left(D\left(A^{*}\right)\right)^{\prime}, L^{2}(\Omega)\right)$, by interpolation we have

$$
\Pi \in \mathcal{L}\left(\mathcal{H}^{-3 / 2+\varepsilon}(\Omega), \mathcal{H}^{1 / 2+\varepsilon}(\Omega)\right) .
$$

Since $\mathcal{H}^{1 / 2+\varepsilon}(\Omega) \subset H^{1 / 2+\varepsilon}(\Omega)$ we obtain

$$
\|\Pi F(z)\|_{H^{1 / 4+\varepsilon / 2}\left(0, \infty ; H^{1 / 2+\varepsilon}(\Omega)\right)} \leq C\|z\|_{H^{1+\varepsilon, 1 / 2+\varepsilon / 2}\left(Q_{\infty}\right)}^{2} .
$$

Then, thanks to Lemma 7.5 , as $H^{1 / 2+\varepsilon}(\Omega) \subset H^{\varepsilon}(\Omega)$, we have

$$
\left\|A_{\Pi}^{-*} \Pi F(z)\right\|_{H^{1 / 4+\varepsilon / 2}\left(0, \infty ; H^{2+\varepsilon}(\Omega)\right)} \leq C\|z\|_{H^{1+\varepsilon, 1 / 2+\varepsilon / 2}\left(Q_{\infty}\right)}^{2} .
$$


Finally, with Proposition 7.1, we obtain

$$
\left\|M B^{*} A_{\Pi}^{-*} \Pi F(z)\right\|_{H^{1 / 4+\varepsilon / 2}\left(0, \infty ; H^{1 / 2+\varepsilon}(\Gamma)\right)} \leq C\|z\|_{H^{1+\varepsilon, 1 / 2+\varepsilon / 2}\left(Q_{\infty}\right)}^{2},
$$

and the proof is complete.

Lemma 7.7. Let $\varepsilon$ belong to $[0,1 / 2)$. We have the following estimate

$\left\|G\left(z_{1}\right)-G\left(z_{2}\right)\right\|_{H^{1 / 4+\varepsilon / 2}\left(0, \infty ; L^{2}(\Omega)\right)} \leq C_{4}\left(\left\|z_{1}\right\|_{H^{1+\varepsilon, 1 / 2+\varepsilon / 2}\left(Q_{\infty}\right)}+\left\|z_{2}\right\|_{H^{1+\varepsilon, 1 / 2+\varepsilon / 2}\left(Q_{\infty}\right)}\right)\left\|z_{1}-z_{2}\right\|_{H^{1+\varepsilon, 1 / 2+\varepsilon / 2}\left(Q_{\infty}\right)}$

for all $z_{1}$ and $z_{2}$ in $H^{1+\varepsilon, 1 / 2+\varepsilon / 2}\left(Q_{\infty}\right)$.

Proof. The estimate in $L^{2}\left(0, \infty ; H^{1 / 2+\varepsilon}(\Gamma)\right)$ can be obtained as in the proof of Lemma 5.5. The estimate in $H^{1 / 4+\varepsilon / 2}\left(0, \infty ; L^{2}(\Gamma)\right)$ follows the lines of Lemma 7.6 . Indeed, we only need to write

$$
\left(1+|\tau|^{2}\right)^{\tilde{\sigma} / 2}\left(1+|\zeta|^{2}\right)^{\tilde{s} / 2}\left(\widehat{z_{1}^{2}}(\tau, \zeta)-\widehat{z_{2}^{2}}(\tau, \zeta)\right)=T_{G}\left(z_{1}-z_{2}, z_{1}+z_{2}\right)(\tilde{\zeta}),
$$

which implies that

$$
\begin{aligned}
\left\|z_{1}^{2}-z_{2}^{2}\right\|_{H^{1 / 4+\varepsilon / 2}\left(0, \infty ; H^{-1 / 2+\varepsilon}(\Omega)\right)} & \leq C\left\|z_{1}+z_{2}\right\|_{H^{3 / 8+\varepsilon / 4}\left(0, \infty ; H^{1 / 4+\varepsilon / 2}(\Omega)\right)}\left\|z_{1}-z_{2}\right\|_{H^{3 / 8+\varepsilon / 4}\left(0, \infty ; H^{1 / 4+\varepsilon / 2}(\Omega)\right)} \\
& \leq C\left(\left\|z_{1}\right\|_{H^{1+\varepsilon, 1 / 2+\varepsilon / 2\left(Q_{\infty}\right)}}+\left\|z_{2}\right\|_{H^{1+\varepsilon, 1 / 2+\varepsilon / 2}\left(Q_{\infty}\right)}\right)\left\|z_{1}-z_{2}\right\|_{H^{1+\varepsilon, 1 / 2+\varepsilon / 2}\left(Q_{\infty}\right)}
\end{aligned}
$$

Then, the proof of Theorem 7.3 is the same as in the case of Neumann boundary conditions. The stabilization result with a prescribed decay rate can be obtained as in Section 6 .

\section{Numerical Simulations}

The computational domain is $\Omega=(0,1) \times(0,1)$. The spatial discretization is carried out using a $P_{1}$ finite element method on a structured triangular mesh. The grid is of size $N=n_{x} \times n_{x}$. The time integration is performed with the implicit scheme given by the MATLAB routine ode $15 \mathrm{~s}$. The control function $u$, acting on the boundary $\Gamma_{1} \subset \Gamma$, is discretized with the same finite element method. Therefore the discretized control is of dimension $n_{x}$.

\subsection{Neumann boundary control}

In this section, we set $\Gamma_{1}=\{1\} \times(0,1)$ and $\Gamma_{2}=\Gamma \backslash \Gamma_{1}$. We want to control the following system

$$
\left\{\begin{array}{l}
\partial_{t} y-\nu \Delta y+y\left(\partial_{1} w+\partial_{2} w\right)+w\left(\partial_{1} y+\partial_{2} y\right)+y\left(\partial_{1} y+\partial_{2} y\right)=0 \quad \text { in } \Omega \times(0, \infty), \\
y(0)=y_{0} \quad \text { in } \Omega \\
\left.\nu \frac{\partial y}{\partial n}\right|_{\Gamma_{1}}=m u \quad \text { and }\left.\quad \nu \frac{\partial y}{\partial n}\right|_{\Gamma_{2}}=0 \quad \text { in }(0, \infty),
\end{array}\right.
$$

where

$$
\nu=\frac{1}{50}, \quad w\left(x_{1}, x_{2}\right)=-0.2 x_{1}, \quad m=1 \text { on } \Gamma_{1},
$$

and $w$ is solution to equation (1.2) with $f\left(x_{1}, x_{2}\right)=0.4 x_{1}$ and $g=-0.2 \nu n_{1}$. The ODE system corresponding to the semi-discretized system is

$$
E^{N} \frac{\mathrm{d} y^{N}(t)}{\mathrm{d} t}=\tilde{A}^{N} y^{N}(t)+\tilde{B}^{N} u^{N}(t)+\tilde{F}^{N}\left(y^{N}(t)\right) \quad \text { in }(0, \infty), \quad y^{N}(0)=y_{0}^{N}
$$


where $E^{N} \in \mathbb{R}^{N \times N}$ is the symmetric mass matrix, $\tilde{A}^{N} \in \mathbb{R}^{N \times N}$ is the stiffness matrix and $\tilde{B} \in \mathbb{R}^{N \times n_{x}}$ is the control matrix. The term $\tilde{F}^{N}\left(y^{N}(t)\right)$ is the discretization in space of the nonlinear term of equation (8.1). We set

$$
A^{N}=\left(E^{N}\right)^{-1} \tilde{A}^{N}, \quad B^{N}=\left(E^{N}\right)^{-1} \tilde{B}^{N}, \quad \text { and } \quad F^{N}\left(y^{N}(t)\right)=\left(E^{N}\right)^{-1} \tilde{F}^{N}\left(y^{N}(t)\right) .
$$

The solution $\Pi^{N}$ of the associated finite dimensional Riccati equation is

$$
\left(A^{N}\right)^{*} \Pi^{N}+\Pi^{N} A^{N}-\Pi^{N} B^{N}\left(B^{N}\right)^{*} \Pi^{N}+E^{N}=0 .
$$

This equation is solved in a classical way, by determining the eigenvectors of the Hamiltonian matrix associated to eigenvalues with negative real parts (see [13]). The linear feedback law is given by

$$
u_{\ell}^{N}=K_{\ell}^{N} y^{N} \quad \text { where } \quad K_{\ell}^{N}=-\left(B^{N}\right)^{*} \Pi^{N},
$$

and the nonlinear control is

$$
u_{n \ell}^{N}=u_{\ell}^{N}+K_{n \ell}^{N} F^{N}\left(y^{N}(t)\right) \quad \text { where } \quad K_{n \ell}^{N}=B^{*}\left(A^{N}-B^{N}\left(B^{N}\right)^{*} \Pi^{N}\right)^{-*} \Pi^{N} .
$$

Remark 8.1. From the expression of the linear operator $(A, D(A))$ associated to system (8.1) we notice that the constant functions are the eigenvectors of $A$ associated to the eigenvalue 0.2. Considering the data (8.2) the matrix $A^{N}$ has only one positive eigenvalue equal to 0.2 .

In the following tests, the Riccati equation (8.4) and the system (8.1) are solved with a grid of size $60 \times 60$ corresponding to 3600 unknowns and 6962 triangles. We compare the efficiency of the linear and the nonlinear feedback laws for various initial conditions. All the uncontrolled solutions are unstable and blow up quickly.

Test 1 . We take a constant perturbation $z_{0}=\delta$. In Figure 1 we have plotted the evolution with time of $|u(t)|_{L^{2}\left(\Gamma_{1}\right)}$ and $|y(t)|_{L^{2}(\Omega)}$.

We see that for $\delta=-0.0625$ the linear law is more efficient than the nonlinear law since for $t \geq 6$ the $L^{2}$-norm of $y$ is smaller. We notice that for $\delta=0.01$ there is no significant difference between the solutions controlled with the linear and the nonlinear control laws. For $\delta=0.05$ the linear law is unable to stabilize the system (8.1) contrary to the nonlinear law, and for $\delta=0.065$ both linear and nonlinear controls are inefficient to stabilize system (8.1).

Test 2. We choose $z_{0}=\delta \sin \left(n \pi x_{1}\right) \cos \left(\ell \pi x_{2}-\phi\right)$ with $\delta>0, n, \ell \in \mathbb{N}^{*}$ and $\phi=0$ or $\pi / 2$. As previously, in Figure 2 we have plotted the evolution with time of the $L^{2}$-norm of the feedback laws and of the solution of equation (8.1). We notice that in each test we have found a value of $\delta$ where the nonlinear law stabilizes the Burgers equation contrary to the linear law.

\subsection{Dirichlet boundary control}

We want to control the following system

$$
\left\{\begin{array}{l}
\partial_{t} y-\nu \Delta y-\alpha y+y\left(\partial_{1} y+\partial_{2} y\right)=0 \quad \text { in } \Omega \times(0, \infty), \\
y(0)=y_{0} \text { in } \Omega, \\
\left.y\right|_{\Gamma_{1}}=m u, \quad \text { and }\left.\quad y\right|_{\Gamma_{2}}=0 \quad \text { in }(0, \infty),
\end{array}\right.
$$

where $\nu=\frac{1}{50}$ and $\alpha>0$. The function $m$ is a smooth function defined by

$$
m(x, \xi)=H\left(\frac{x}{\xi}-1\right)-H\left(\frac{(x-1)}{\xi}+1\right), \quad \xi>0, \quad x \in \mathbb{R},
$$



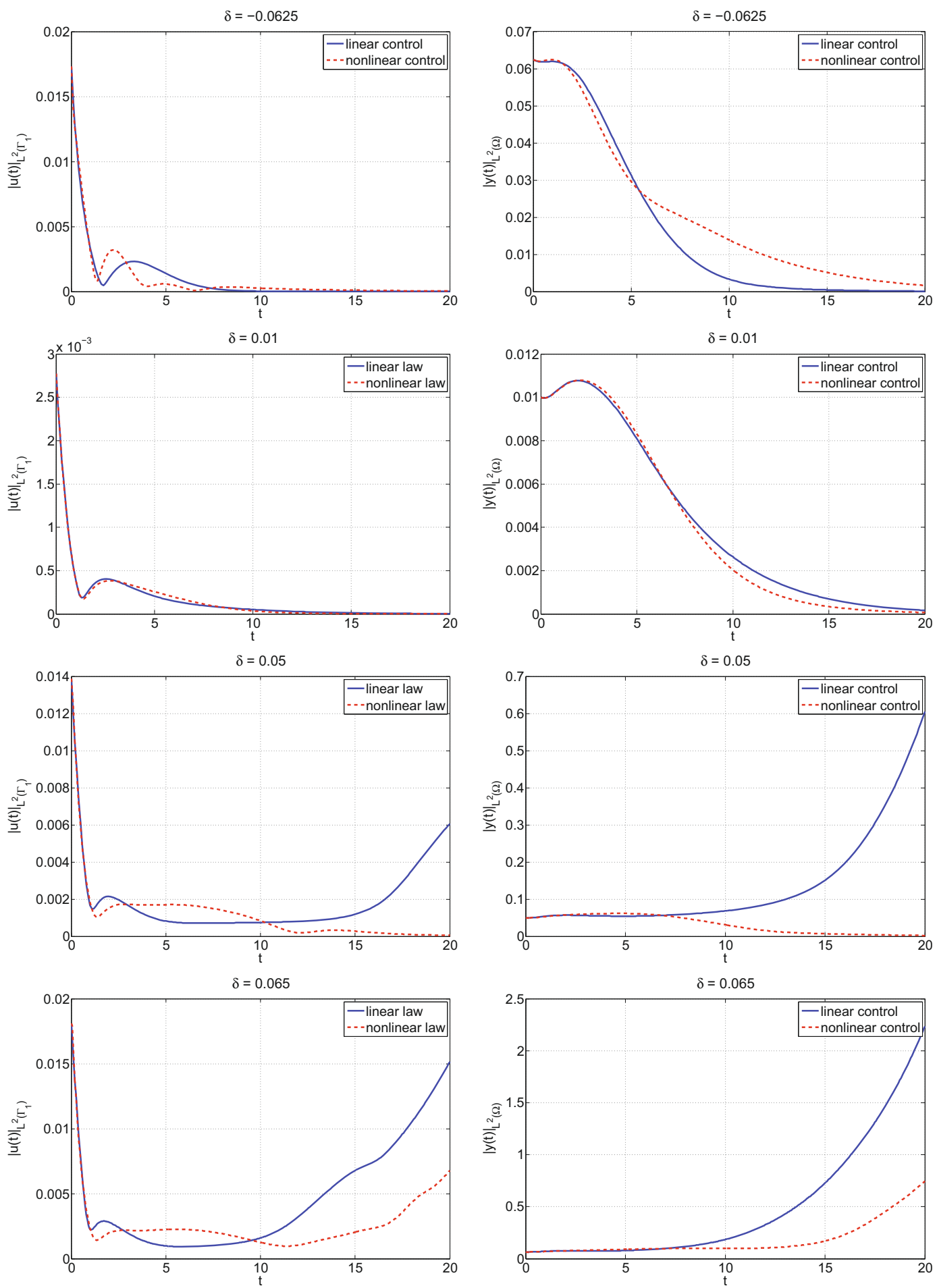

FiguRE 1. Evolution with time of $|u(t)|_{L^{2}\left(\Gamma_{1}\right)}$ and $|y(t)|_{L^{2}(\Omega)}$ controlled with linear and nonlinear laws with $z_{0}=\delta$. 

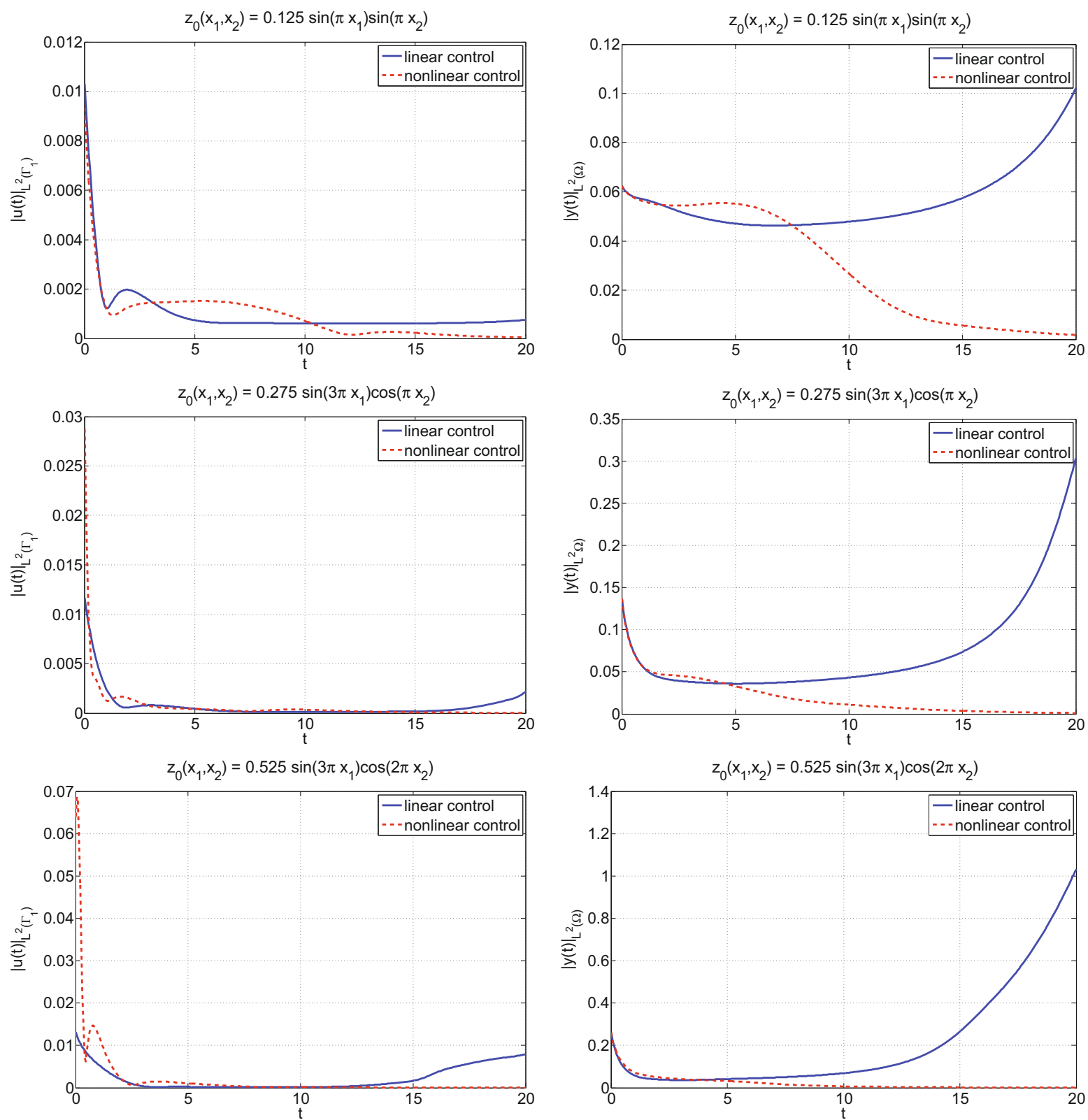

Figure 2. Evolution with time of $|u(t)|_{L^{2}\left(\Gamma_{1}\right)}$ (left) and $|y(t)|_{L^{2}(\Omega)}$ (right) controlled with linear and nonlinear laws with $z_{0}\left(x_{1}, x_{2}\right)=0.125 \sin \left(\pi x_{1}\right) \sin \left(\pi x_{2}\right), z_{0}\left(x_{1}, x_{2}\right)=$ $0.275 \sin \left(3 \pi x_{1}\right) \cos \left(\pi x_{2}\right)$ and $z_{0}\left(x_{1}, x_{2}\right)=0.525 \sin \left(3 \pi x_{1}\right) \cos \left(2 \pi x_{2}\right)$.

where

$$
H(x)=\left\{\begin{array}{l}
0 \quad \text { if } x \leq-1, \\
0.5+x\left(0.9375-x^{2}\left(0.625-0.1875 x^{2}\right)\right) \quad \text { if }-1<x<1, \\
1 \quad \text { if } x \geq 1 .
\end{array}\right.
$$




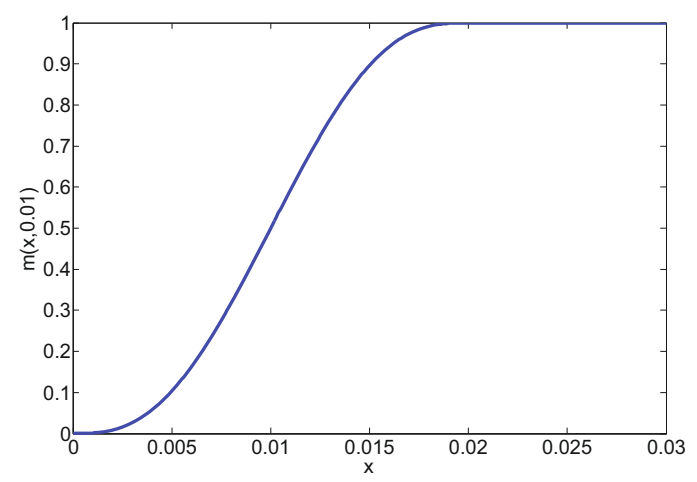

Figure 3. Graph of $m(x, 0.01)$ for $x \in[0,0.03]$.

In the numerical simulations we set $\xi=0.01$. The graph of the function $m$ for $x \in[0,0.03]$ and $\xi=0.01$ is given in Figure 3.

To implement the inhomogeneous Dirichlet boundary condition on $\Gamma_{1}$ we use a Robin condition of the form

$$
\left.\nu \frac{\partial y}{\partial n}\right|_{\Gamma_{1}}=-\frac{1}{\varepsilon}(y-m u) \quad \text { with } \quad \varepsilon=10^{-4} .
$$

This condition is very well adapted to take into account numerically the inhomogeneous Dirichlet condition [4] and in particular the nonlinear feedback law. The eigenvalues of the operator $A y=\nu \Delta y+\alpha y$ with $y=0$ on $\Gamma$ are

The corresponding eigenvectors are

$$
\lambda_{i j}=-\nu\left(i^{2}+j^{2}\right) \pi^{2}+\alpha, \quad i, j \in \mathbb{N}^{*} .
$$

$$
\varphi_{i j}\left(x_{1}, x_{2}\right)=\delta \sin \left(i \pi x_{1}\right) \sin \left(j \pi x_{2}\right), \quad \delta \in \mathbb{R}^{*} .
$$

In the following tests, the parameter $\alpha$ is equal to 0.6 . With this choice the operator $A$ has one positive eigenvalue which is $\lambda_{11} \approx 0.2052$.

We are going to see that we have different behaviors of controlled solutions depending on the choice of $\Gamma_{1}$. In Test 1 , we choose $\Gamma_{1}=\{0\} \times(0,1)$ and in Test 2 and Test 3 we choose $\Gamma_{1}=\{1\} \times(0,1)$. In all cases, we set $\Gamma_{2}=\Gamma \backslash \Gamma_{1}$.

Test 1. The initial data is chosen as follows

$$
y_{0}\left(x_{1}, x_{2}\right)=0.045 \sin \left(\pi x_{1}\right) \sin \left(\pi x_{2}\right) \quad \text { with } \quad\left(x_{1}, x_{2}\right) \in[0,1] \times[0,1] .
$$

In Figure 4, we have plotted the $L^{2}$-norm of $y$ (left) and the $L^{2}$-norm of $\nabla y$ (right) with respect to $t$. We remark that after a transient stage, the $L^{2}$-norm of $y(t)$ and $\nabla y(t)$ are smaller with the nonlinear feedback law. Figure 5 shows that, during the first seconds, the nonlinear control is stronger than the linear one.

Test 2. The initial data is chosen as follows

$$
y_{0}\left(x_{1}, x_{2}\right)=0.2 \sin \left(\pi x_{1}\right) \sin \left(\pi x_{2}\right) \quad \text { with } \quad\left(x_{1}, x_{2}\right) \in[0,1] \times[0,1] .
$$

As previously we have plotted in Figure 6 the $L^{2}$-norm of $y$ (left) and the $L^{2}$-norm of $\nabla y$ (right) with respect to $t$. The results are now different. By applying the control laws on the right boundary of the domain, the linear law becomes more performant than the nonlinear one. In Figure 7 we remark that the linear control is now stronger during the first seconds than the nonlinear one. 

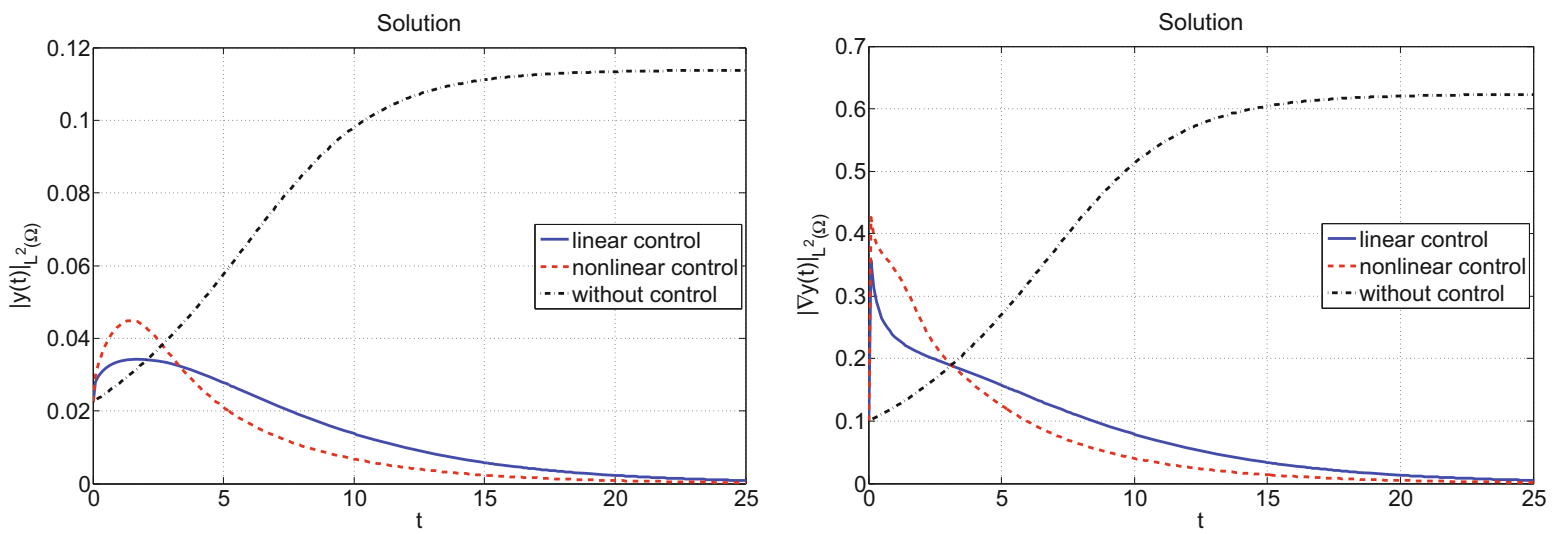

Figure 4. Evolution with time of $|y(t)|_{L^{2}(\Omega)}$ (left) and $|\nabla y(t)|_{L^{2}(\Omega)}$ (right) uncontrolled and controlled with linear and nonlinear laws.

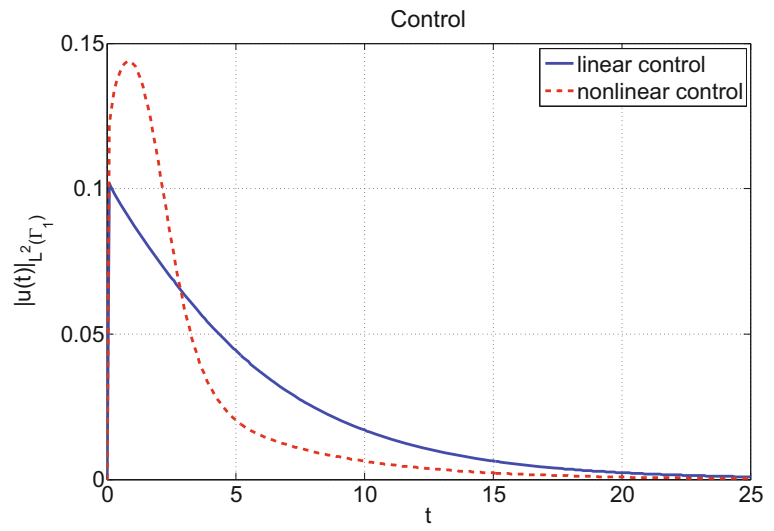

FIGURE 5. Evolution with time of $\left|u_{\ell}(t)\right|_{L^{2}\left(\Gamma_{1}\right)}$ and $\left|u_{n \ell}(t)\right|_{L^{2}\left(\Gamma_{1}\right)}$.
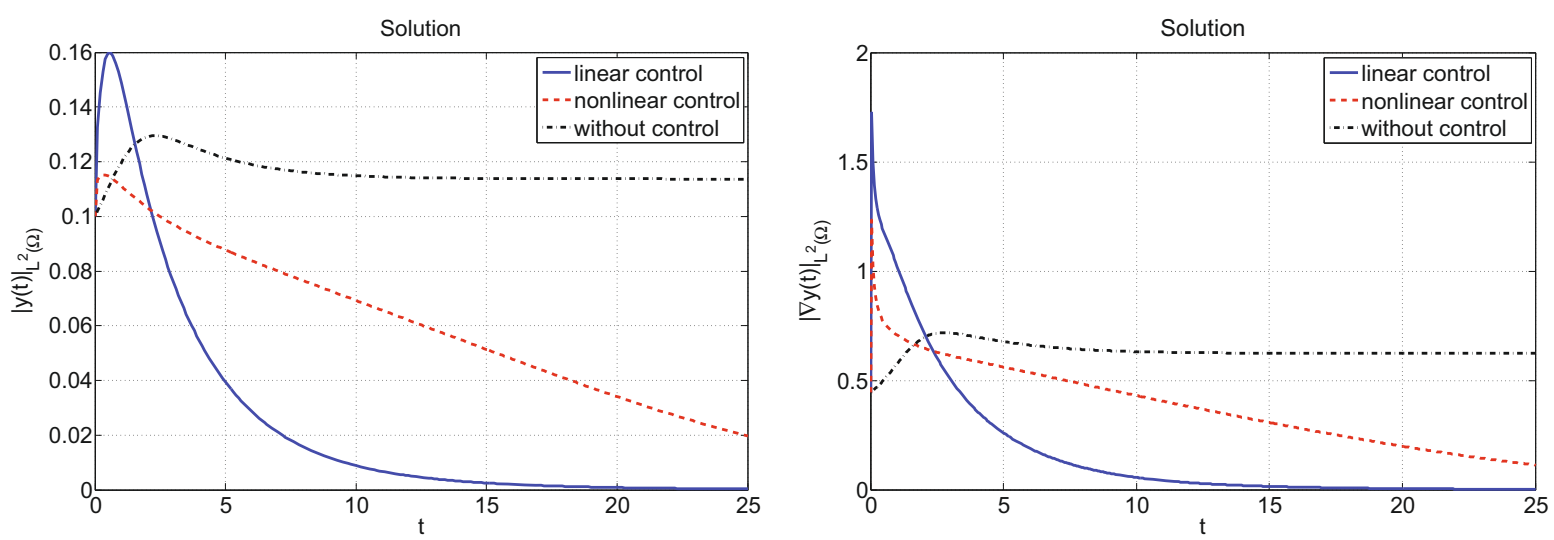

Figure 6. Evolution with time of $|y(t)|_{L^{2}(\Omega)}$ (left) and $|\nabla y(t)|_{L^{2}(\Omega)}$ (right) uncontrolled and controlled with linear and nonlinear laws. 


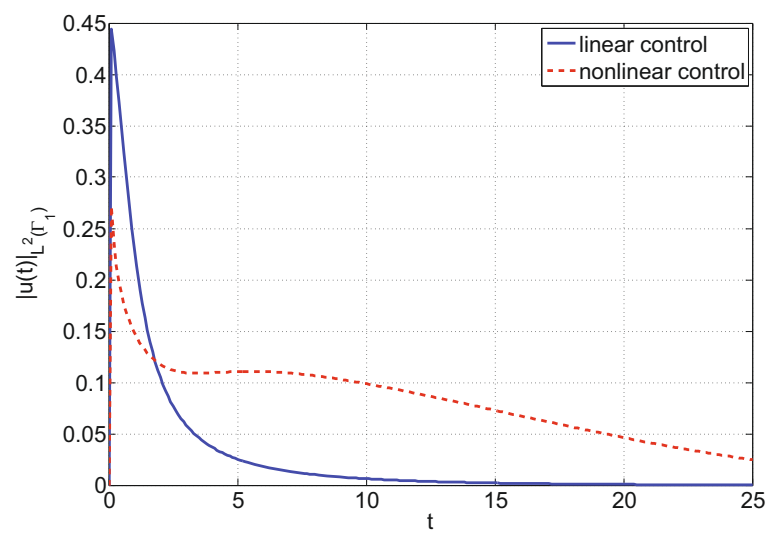

FiguRE 7. Evolution with time of $\left|u_{\ell}(t)\right|_{L^{2}\left(\Gamma_{1}\right)}$ and $\left|u_{n \ell}(t)\right|_{L^{2}\left(\Gamma_{1}\right)}$.
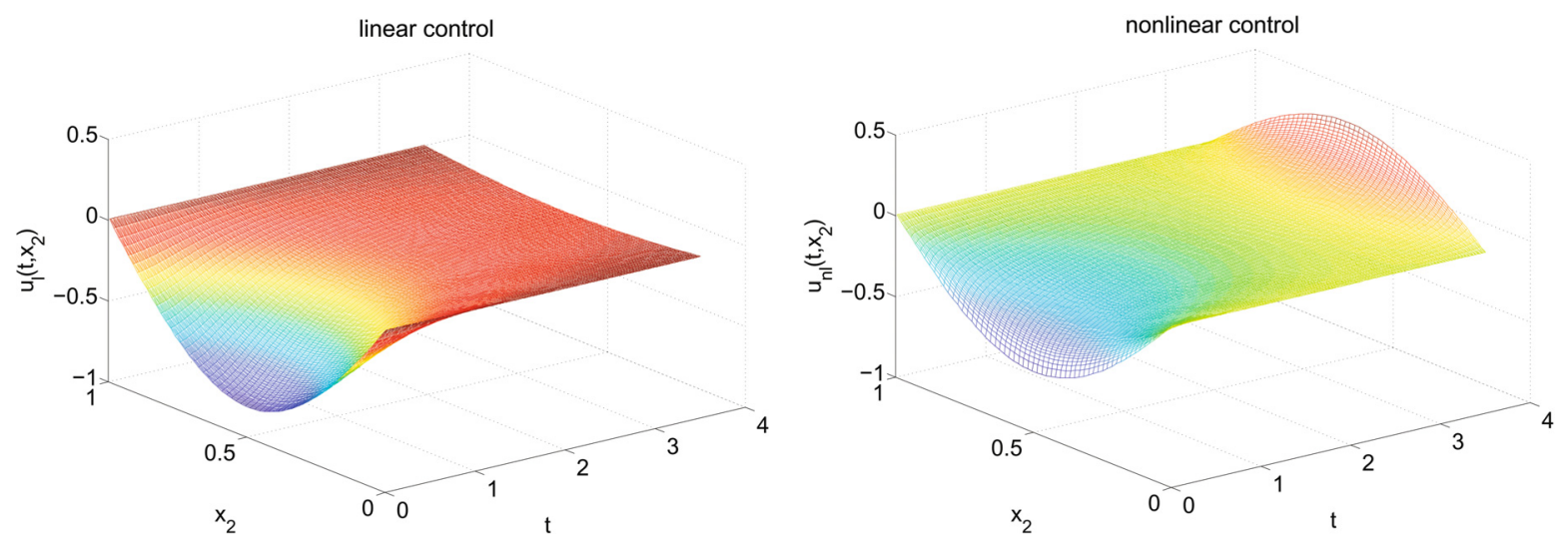

FIGURE 8. Graphs of $u_{\ell}\left(t, x_{2}\right)$ (left) and $u_{n \ell}\left(t, x_{2}\right)$ (right) for $\left(t, x_{2}\right) \in[0.01,3.5] \times[0,1]$.

Test 3. In the last test, we increase the $L^{\infty}-$ norm of the initial condition by taking

$$
y_{0}\left(x_{1}, x_{2}\right)=0.25 \sin \left(\pi x_{1}\right) \sin \left(\pi x_{2}\right) \quad \text { with } \quad\left(x_{1}, x_{2}\right) \in[0,1] \times[0,1] .
$$

The simulations are stopped for $t=3.5$. In Figures 8 and 9 we notice that the nonlinear law grows up quickly after $t=2.5$. To explain more precisely these graphs, we have plotted the $L^{2}$-norm of the linear part and the nonlinear part of the nonlinear law (8.5) in Figure 10. The nonlinear term has an opposite sign to the linear one and becomes too large after $t=2.5$. It destabilizes the Burgers equation.

Remark 8.2. We have tested various values of $\delta$ for initial conditions of the form

$$
y_{0}\left(x_{1}, x_{2}\right)=\delta \sin \left(\pi x_{1}\right) \sin \left(\pi x_{2}\right)
$$

In all simulations, the linear law acts stronger than the nonlinear one. For some values of $\delta$, the nonlinear law is unable to stabilize the Burgers equation. The main advantage of the nonlinear law is that it limits the overshoots during the first time steps. Therefore it seems more adapted in the real experimental configurations. To improve the stabilization result, it should be interesting to build a feedback law defined with an appropriate 

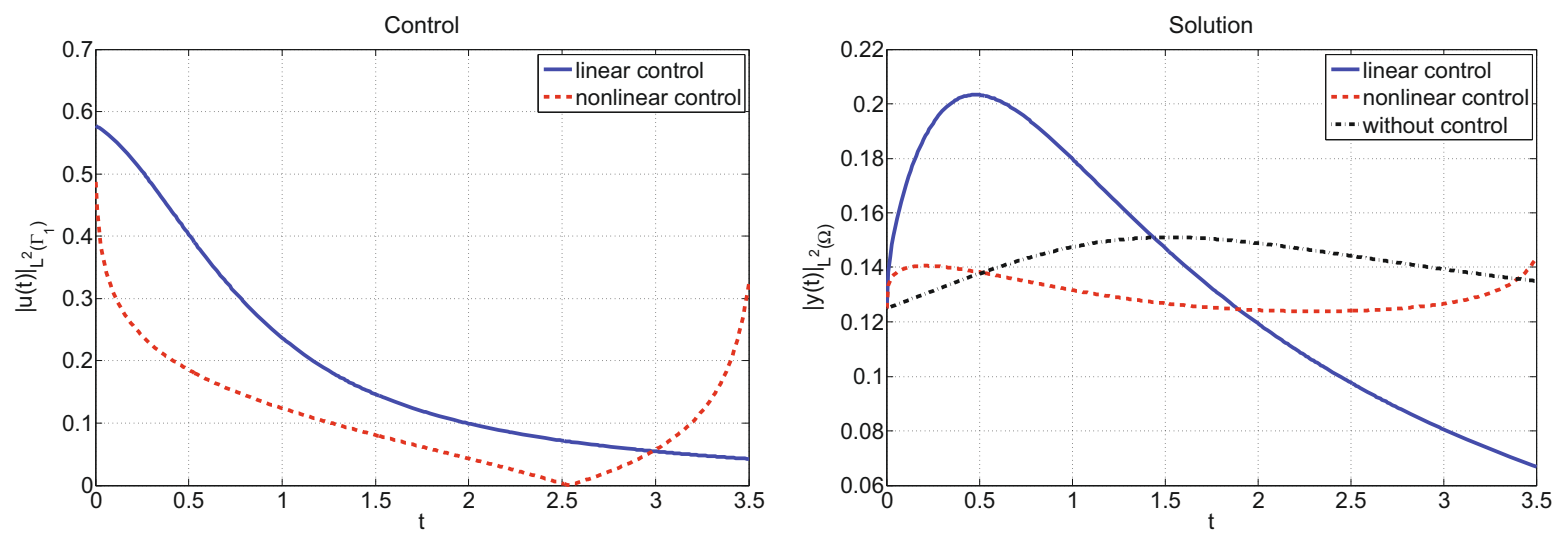

Figure 9. Evolution with time of $\left|u_{\ell}(t)\right|_{L^{2}\left(\Gamma_{1}\right)},\left|u_{n \ell}(t)\right|_{L^{2}\left(\Gamma_{1}\right)}$ (left) and $|y(t)|_{L^{2}(\Omega)}$ (right) uncontrolled and controlled with linear and nonlinear laws.

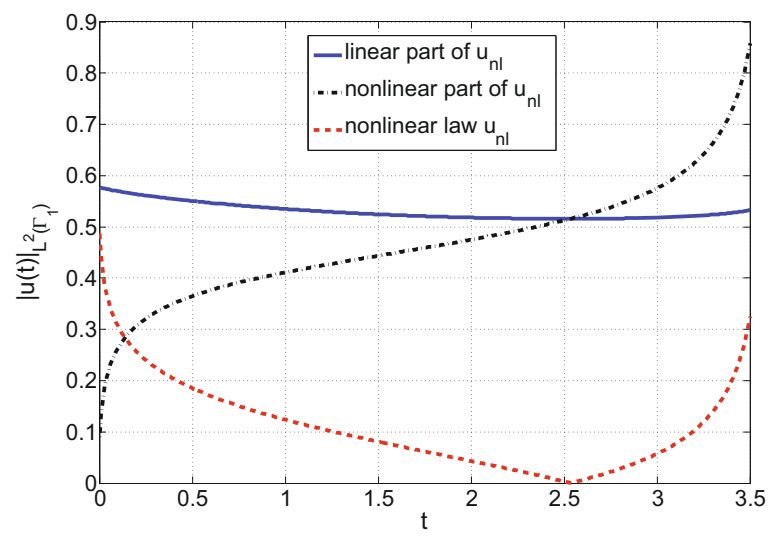

FiguRE 10. Evolution with time of $L^{2}$-norm of the linear part and the nonlinear part of the nonlinear law (8.5).

combination of the linear and the nonlinear laws. For that it is necessary to find Lyapunov functions for the closed loop nonlinear systems. We think that the results in $[1,2]$ can be very helpful for such an analysis.

\section{REFERENCES}

[1] M. Badra, Stabilisation par feedback et approximation des équations de Navier-Stokes. Ph.D. Thesis, Université Paul Sabatier, Toulouse, France (2006).

[2] M. Badra, Lyapunov function and local feedback boundary stabilization of the Navier-Stokes equations. SIAM J. Control. (to appear).

[3] S.C. Beeler, H.T. Tran and H.T. Banks, Feedback control methodologies for nonlinear systems. J. Optim. Theory Appl. 107 (2000) 1-33.

[4] F. Ben Belgacem, H. El Fekik and J.-P. Raymond, A penalized Robin approach for solving a parabolic equation with non smooth Dirichlet boundary conditions. Asymptotic Anal. 34 (2003) 121-136.

[5] A. Bensoussan, G. Da Prato, M.C. Delfour and S.K. Mitter, Representation and Control of Infinite Dimensional Systems, Vol. 1. Birkhäuser (1992). 
[6] A. Bensoussan, G. Da Prato, M.C. Delfour and S.K. Mitter, Representation and Control of Infinite Dimensional Systems, Vol. 2. Birkhäuser (1993).

[7] E. Fernandez-Cara, S. Guerrero, O. Yu. Imanuvilov and J.-P. Puel, Local exact controllability of the Navier-Stokes system. J. Math. Pures Appl. 83 (2004) 1501-1542.

[8] E. Fernandez-Cara, M. Gonzalez-Burgos, S. Guerrero and J.-P. Puel, Exact controllability to the trajectories of the heat equation with Fourier boundary conditions: the semilinear case. ESAIM: COCV 12 (2006) 466-483 (electronic).

[9] G. Grubb and V.A. Solonnikov, Boundary value problems for the nonstationary Navier-Stokes equations treated by pseudodifferential methods. Math. Scand. 69 (1991) 217-290.

[10] L. Hormander, Lectures on Nonlinear Hyperbolic Differential Equations. Springer (1997).

[11] M. Krstic, L. Magnis and R. Vazquez, Nonlinear stabilization of shock-like unstable equilibria in the viscous Burgers PDE. IEEE Trans. Automat. Contr. 53 (2008) 1678-1683.

[12] I. Lasiecka and R. Triggiani, Control Theory for Partial Differential Equations, Vol. 1. Cambridge University Press (2000).

[13] A.J. Laub, A Schur method method for solving algebraic Riccati equations. IEEE Trans. Automat. Contr. 24 (1979) 913-921.

[14] J.-L. Lions, Espaces d'interpolation et domaines de puissances fractionnaires d'opérateurs. J. Math. Soc. Japan 14 (1962) 233-241.

[15] J.-L. Lions and E. Magenes, Problèmes aux limites non homogènes, Vol. 2. Dunod, Paris (1968).

[16] J.-P. Raymond, Boundary feedback stabilization of the two dimensional Navier-Stokes equations. SIAM J. Control Optim. 45 (2006) 790-828. 